\title{
A New Uncertain Analysis Method for the Prediction of Acoustic Field with Random and Interval Parameters
}

\author{
Mingjie Wang, ${ }^{1}$ Zhimin Wan, $^{2}$ and Qibai Huang ${ }^{1}$ \\ ${ }^{1}$ State Key Laboratory of Digital Manufacturing Equipment and Technology, Huazhong University of Science and Technology, \\ Wuhan 430074, China \\ ${ }^{2}$ College of Automobile and Transportation Engineering, Nantong Vocational University, Nantong 226000, China
}

Correspondence should be addressed to Qibai Huang; qbhuang@hust.edu.cn

Received 26 January 2016; Accepted 7 April 2016

Academic Editor: Alba Sofi

Copyright (C) 2016 Mingjie Wang et al. This is an open access article distributed under the Creative Commons Attribution License, which permits unrestricted use, distribution, and reproduction in any medium, provided the original work is properly cited.

For the frequency response analysis of acoustic field with random and interval parameters, a nonintrusive uncertain analysis method named Polynomial Chaos Response Surface (PCRS) method is proposed. In the proposed method, the polynomial chaos expansion method is employed to deal with the random parameters, and the response surface method is used to handle the interval parameters. The PCRS method does not require efforts to modify model equations due to its nonintrusive characteristic. By means of the PCRS combined with the existing interval analysis method, the lower and upper bounds of expectation, variance, and probability density function of the frequency response can be efficiently evaluated. Two numerical examples are conducted to validate the accuracy and efficiency of the approach. The results show that the PCRS method is more efficient compared to the direct Monte Carlo simulation (MCS) method based on the original numerical model without causing significant loss of accuracy.

\section{Introduction}

It is well known that the numerical method for the frequency response analysis of the acoustic field with determined parameters has achieved great attention. However, a complicated acoustic field contains many uncertain factors, such as the model inaccuracies produced by the manufacturing tolerance and measuring error, the admittance coefficients variation induced by the inhomogeneity of absorber materials, and the unpredictability of environment. Hence, the response of an acoustic field is always subjected to these uncertain parameters. The main approaches to model these uncertainties are the probabilistic methods and the nonprobabilistic methods. In the probabilistic methods, uncertain parameters can be modeled as random variables through the description of predefined probability distribution functions. Among these probabilistic methods, the MCS method $[1,2]$ is widely used due to the ease of implementation, while its main shortcoming is time consuming to obtain accurate results. Therefore, the MCS method is usually used as an approach for validating the accuracy and efficiency of other methods. Perturbation stochastic method is an alternative approach for the probabilistic problems $[3,4]$. In this method, both input and output structural parameters are expanded using Taylor series with random parameters. However, this approach may be only suitable for small uncertainty level of parameters. Spectral stochastic method is also an efficient alternative for stochastic problems. The basic idea of the spectral stochastic method is to employ a series expansion to model the relationship between the input uncertainty and the output variability. The polynomial chaos expansion, one of the spectral stochastic methods, has been previously applied in uncertainty propagation in various fields, such as structural dynamics [5], fluid dynamics [6], and acoustic fields [7-10]. Sarkar and Ghanem [5] introduced the polynomial chaos expansion method to study the midfrequency vibration of linear random systems with random parameters. Sepahvand et al. [7] applied the generalized polynomial chaos to study the vibroacoustic performance of laminated composite plates with uncertain elastic parameters. LePage [8] adopted the polynomial chaos expansion method to investigate sonar system performance in uncertain environments. The simulation results agree very closely with the results estimated by MCS with less computational involvement. The polynomial chaos expansion 
method is suitable for studying the uncertainty propagation of systems with uncertainties in model parameters, initial conditions, or inputs [11]. More information about the advantages of the polynomial chaos expansion over other methods, such as MCS and perturbation techniques, in uncertainty modeling can be found in [12-16]. From the overall perspective, probabilistic methods have achieved great success in predicting the statistic information of system response, when the probability density functions (PDFs) of uncertain parameters are available. Unfortunately, in many practical applications, it is hard or costly to construct the probability density distributions of uncertain parameters.

For the system with limited information, the nonprobabilistic methods are the suitable alternatives. Interval analysis, one of the nonprobabilistic methods, has attracted widespread attention. The fundamentals on the interval mathematics were first introduced in [17]. After the pioneering work of Alefeld and Herzberger [18], various interval methods $[19,20]$ have been developed to solve static structural systems with interval parameters. The lower and upper bounds of response can be calculated directly using the interval mathematics. The main drawback of these methods is the overestimation caused by the wrapping effect. Reducing overestimation has been an active area of research and the related articles can be found in [21-23]. Xia and Yu [21] proposed a modified interval perturbation finite element method for the frequency response analysis of the structural-acoustic system with interval parameters. Xia et al. [22] introduced a subinterval perturbation finite element method to predict the structural-acoustic field with large uncertain-but-bounded parameters. The numerical results of these methods verify the effectiveness on reducing the overestimation, but these methods require more efforts on modifying the governing equations. Recently, Fang et al. [24] developed a new interval response surface model for the purpose of efficiently implementing the interval model updating procedure. In this method, a simple interval surrogate model was built based on traditional response surface method, and the response intervals can be calculated efficiently. Zou et al. [25] proposed a method by combining MCS method and response surface method to study the uncertainty propagation in accident reconstruction.

As mentioned above, most researches on uncertain problems have been carried out by using either interval model or random model. However, the real uncertain engineering problems involve the random parameters and interval parameters simultaneously. Thus, it is desirable to construct a hybrid uncertain method to handle the system with hybrid parameters. Guo and Du [26] proposed a sensitivity analysis method for a mixture of random and interval variables. Gao et al. [27] developed a mixed perturbation Monte Carlo method (HPMCM) for static and reliability analysis of the structural systems with hybrid parameters. To reduce the excessive computational efforts of HPMCM suffering from MCS, a hybrid perturbation vertex method (HPVM) for the frequency response analysis of hybrid uncertain acoustic field was proposed by Xia et al. [28]. However, the simulation results of HPVM cannot be accurate when the extreme values of the response are not at the vertexes.
For the numerical analysis of the uncertain acoustic field and the structural-acoustic field, a large number of uncertain methods have been developed. According to the approaches to describe the uncertain parameters, the uncertain acoustic model is mainly divided into the probabilistic model, the interval model, the convex model, and the two-type hybrid uncertain models. For the response analysis of acoustic field with random parameters, a probabilistic method named as the change-of-variable perturbation stochastic finite element method has been proposed by Xia and Yu [29]. For the response analysis of the acoustic field with interval parameters, two interval analysis methods named as the interval perturbation method and the modified interval perturbation method have been proposed [22, 30-32]. For the response analysis of acoustic field with convex parameters, two convex methods named as first-order convex perturbation method and second-order convex perturbation method have been proposed by Xia and $\mathrm{Yu}$ [33]. For the response analysis of acoustic field with both random and interval parameters, two numerical methods named as the hybrid perturbation Monte Carlo method and the hybrid perturbation vertex method have been proposed by Xia et al. [28, 34]. For the response analysis of acoustic field with interval random parameters, Xia et al. [35, 36] proposed an interval random model in which the uncertain parameters are quantified as the random variables, while some of the probability distribution parameters of random variables can only be provided with the variation intervals but not the precise values.

From the overall perspective, current research on uncertain acoustic field is mainly based on perturbation theory, which requires additional efforts on the deduction of mathematical formula. To overcome such inconveniences, nonintrusive methods can be attractive alternatives. However, up to now, to the best of the authors' knowledge, the relevant research on the nonintrusive methods for response analysis of the acoustic field with random parameters and interval parameters is still not reported. In this work, our efforts are concentrated specifically on developing a nonintrusive uncertain analysis method for acoustic systems with hybrid parameters. Firstly, a nonintrusive polynomial chaos expansion method is employed to handle the random parameters due to its easiness to recover information and characterize the variability, in particular the mean and the variance. Then, the response surface method is used to deal with the interval parameters because of its simplicity and ease of use. Finally, the upper and lower bounds of the probabilistic characteristics can be calculated by means of the existing interval analysis methods.

The organization of this paper is as follows. In Section 2, the dynamic equation of the acoustic system with random parameters and interval parameters is derived. In Section 3, the polynomial chaos expansion method for random variables is presented. In Section 4, the response surface method for interval variables is presented. In Section 5, the proposed PCRS is constructed for frequency response analysis of the acoustic system with random variables and interval variables. In Section 6, two acoustic models with random and interval parameters are conducted to verify the effectiveness of 
the proposed methods. Several conclusions drawn based on the current study are given in the last section.

\section{Acoustic FE Model with Random and Interval Variables}

Consider an acoustic cavity of volume $\Omega$, whose boundary $\Gamma$ of the acoustic cavity $\Omega$ is composed of three parts. These are the Dirichlet boundary condition $\Gamma_{D}$, the Neumann boundary condition $\Gamma_{N}$, and the Robin boundary condition $\Gamma_{R}$, respectively.

Assuming that the state of the acoustic field is steady, the sound pressure $p$ is governed by the Helmholtz equation:

$$
\nabla^{2} p+k^{2} p=0
$$

where $\nabla^{2}$ is the Laplace operator, $k=w / c$ is the wave number, $w$ is the angular frequency, and $c$ is the sound speed. The three types of boundary condition can be expressed as

$$
\begin{gathered}
p=\bar{p} \quad \text { on } \Gamma_{D}, \\
\frac{\partial p}{\partial \mathbf{n}}=-j \rho w v_{n} \quad \text { on } \Gamma_{N}, \\
\frac{\partial p}{\partial \mathbf{n}}=-j \rho w A p \quad \text { on } \Gamma_{R},
\end{gathered}
$$

where $\bar{p}$ is the imposed sound pressure on boundary $\Gamma_{D}, \mathbf{n}$ is the exterior unit-normal vector, $j=\sqrt{-1}$ is an imaginary unit, $\rho$ is the density of the fluid in acoustic field, $v_{n}$ is the normal velocity imposed on boundary $\Gamma_{N}$, and $A$ is the admittance coefficient of the damping material on boundary $\Gamma_{R}$.

In the standard finite element analysis, the acoustic domain $\Omega$ is discretized into a number of acoustic elements. The governing equation of the acoustic field in finite element framework can be written as

$$
\left(\mathbf{K}+j w \mathbf{C}-w^{2} \mathbf{M}\right) \mathbf{p}=\mathbf{q},
$$

where $\mathbf{K}, \mathbf{M}, \mathbf{C}$, and $\mathbf{q}$ represent the acoustic system stiffness matrix, mass matrix, damping matrix, and load vector, respectively. They can be expressed as follows:

$$
\begin{aligned}
& \mathbf{K}=\sum_{n_{e}} \int_{\Omega_{e}}(\nabla \mathbf{N})^{T} \nabla \mathbf{N} d \Omega_{e}, \\
& \mathbf{M}=\sum_{n_{e}} \int_{\Omega_{e}} \frac{1}{c^{2}} \mathbf{N}^{T} \mathbf{N} d \Omega_{e}, \\
& \mathbf{C}=\sum_{n_{e}} \int_{\Gamma_{R}^{e}} \rho A_{e} \mathbf{N}^{T} \mathbf{N} d \Gamma, \\
& \mathbf{q}=j w \rho \sum_{n_{e}} \int_{\Omega_{e}} \mathbf{N}^{T} q_{a} d \Omega_{e},
\end{aligned}
$$

where $\mathbf{N}$ denotes the element shape function, the subscript $e$ after $\Omega, \Gamma$, and $A$ represents the corresponding quantity for a finite element, and $q_{a}$ is the volume velocity per unit volume.
The governing equation in acoustic field can be written as

$$
\mathbf{Z P}=\mathbf{q},
$$

where $\mathbf{Z}$ denotes the acoustic dynamic stiffness matrix, which can be expressed as

$$
\mathbf{Z}=\left(\mathbf{K}+j w \mathbf{C}-w^{2} \mathbf{M}\right) .
$$

For actual acoustic problem, uncertainties in material properties, external loads, and geometric dimensions are unavoidable due to the manufacturing errors and the measured errors. Assume that the uncertain parameters of the acoustic cavity are independent. The uncertain parameters whose probability distributions are known can be modeled as random variables. The uncertain parameters with limited information can be described as the interval variables. Then the finite element equation of the acoustic field with both random and interval variables can be written as

$$
\begin{aligned}
\mathrm{Z}(\xi, \boldsymbol{\eta}) \mathbf{P}(\xi, \boldsymbol{\eta}) & =\mathbf{q}(\xi, \boldsymbol{\eta}), \\
\mathbf{P}(\xi, \boldsymbol{\eta}) & =\mathrm{Z}^{-1}(\boldsymbol{\xi}, \boldsymbol{\eta}) \mathbf{q}(\xi, \boldsymbol{\eta}),
\end{aligned}
$$

where $\boldsymbol{\xi}=\left(\xi_{1}, \xi_{2}, \ldots, \xi_{n}\right)$ denotes the random vector and $\boldsymbol{\eta}=$ $\left(\eta_{1}, \eta_{2}, \ldots, \eta_{m}\right)$ is the interval vector. From (7), it can be seen that the frequency response $\mathbf{P}$ is the function of both random vector $\boldsymbol{\xi}$ and interval vector $\boldsymbol{\eta}$.

\section{The Polynomial Chaos Expansion Method for Random Variables}

The basic idea of the polynomial chaos expansion method is that any random process can be approximated by a linear combination of orthogonal polynomials of independent random variables [7]. Wiener [37] first introduced the polynomial chaos in the form of homogeneous expansion using orthogonal Hermite polynomial basis. The approach has been extended to the generalized polynomial chaos [14] to deal with more general random fields such as multidimensional random fields and non-Gaussian random fields.

In this section, we assume that the acoustic system only contains random variables. If the input parameters are described as the uniformly distributed random variables, the frequency response $P$ can be expressed by an expansion of multidimensional Legendre polynomials function of the input vector $\xi=\left(\xi_{1}, \ldots, \xi_{n}\right)$. For other types of random variable, different polynomial bases can be found in [14]. Considering the uniformly distributed random variable, the frequency response can be expressed as follows:

$$
P(\boldsymbol{\xi})=\sum_{k=0}^{\infty} u_{k} \psi_{k}(\boldsymbol{\xi}),
$$

where $u_{k}$ represents the deterministic coefficient to be estimated and $\psi_{k}(\xi)$ is the Legendre polynomial chaos of order $k$. Naturally, for the purposes of numerical computation, the terms given by (9) must be truncated. Assuming that the number of the truncated terms is $s, P(\xi)$ can be written as

$$
P(\xi)=\sum_{k=0}^{s-1} u_{k} \psi_{k}(\xi)
$$


The number of the truncated terms $s$ depends on the number of random dimensions $(n)$ of $\xi$ and the highest order $(\gamma)$ of the polynomials $\psi$, which is given by

$$
s=\frac{(n+\gamma) !}{n ! \gamma !}
$$

Considering the one-dimensional Legendre polynomial, the three-term recurrence relation can be given by

$$
\begin{aligned}
H_{0}(\xi) & =1, \\
H_{1}(\xi) & =\xi \\
H_{j+1}(\xi) & =\frac{2 j+1}{j+1} \xi H_{j}(\xi)-\frac{j}{j+1} H_{j-1}(\xi), \\
& (j=1, \ldots, n),
\end{aligned}
$$

where $j$ denotes the order of Legendre polynomials. For example, the one-dimensional Legendre polynomials are

$$
\begin{aligned}
& \psi_{0}=1, \\
& \psi_{1}=\xi \\
& \psi_{2}=\frac{3}{2} \xi^{2}-\frac{1}{2}, \\
& \psi_{3}=\frac{5}{2} \xi^{3}-\frac{3}{2} \xi, \ldots
\end{aligned}
$$

For multidimensional random variable $\xi=\left(\xi_{1}, \ldots, \xi_{n}\right)$, the Legendre polynomials can be expressed as follows:

$$
\begin{aligned}
H_{m_{1}, m_{2}, \ldots, m_{n}}\left(\xi_{1}, \ldots, \xi_{n}\right)=\prod_{k=1}^{n} H_{m_{k}}\left(\xi_{k}\right), & \\
& \left(m_{k}=0,1, \ldots, \gamma\right) .
\end{aligned}
$$

Then, the two-dimensional Legendre polynomials are given by

$$
\begin{gathered}
\psi_{0}=H_{0,0}\left(\xi_{1}, \xi_{2}\right)=1, \\
\psi_{1}=H_{1,0}\left(\xi_{1}, \xi_{2}\right)=\xi_{1}, \\
\psi_{2}=H_{0,1}\left(\xi_{1}, \xi_{2}\right)=\xi_{2}, \\
\psi_{3}=H_{2,0}\left(\xi_{1}, \xi_{2}\right)=\frac{3}{2} \xi_{1}^{2}-\frac{1}{2}, \\
\psi_{4}=H_{1,1}\left(\xi_{1}, \xi_{2}\right)=\xi_{1} \xi_{2}, \\
\psi_{5}=H_{0,2}\left(\xi_{1}, \xi_{2}\right)=\frac{3}{2} \xi_{2}^{2}-\frac{1}{2}
\end{gathered}
$$

The orthogonality relation of the Legendre polynomial chaos takes the form

$$
\left\langle\psi_{i}, \psi_{j}\right\rangle=\left\langle\psi_{i}^{2}\right\rangle \delta_{i j}
$$

where $\delta_{i j}$ is the Kronecker delta and $\langle\cdot, \cdot\rangle$ denotes the ensemble average:

$$
\langle f, g\rangle=\int f(\xi) g(\xi) w(\xi) d \xi .
$$

$w(\xi)$ is the joint probability density of the random variable $\boldsymbol{\xi}$ [38]. The coefficients $u_{k}$ in (10) can be obtained as follows:

$$
u_{k}=\frac{\left\langle P(\boldsymbol{\xi}), \psi_{k}\right\rangle}{\left\langle\psi_{k}^{2}\right\rangle}=\frac{1}{\left\langle\psi_{k}^{2}\right\rangle} \int P(\boldsymbol{\xi}) \psi_{k}(\boldsymbol{\xi}) w(\boldsymbol{\xi}) d \boldsymbol{\xi} .
$$

There are two broad approaches to calculating these coefficients: the intrusive approach and the nonintrusive approach. The intrusive approach requires the complete reformulation of any existing code, which is often difficult and prohibitively costly for complex systems. Thus, the nonintrusive approach is an attractive alternative, where we do not require changes to deterministic solvers for $P$ as we generate realizations of $\boldsymbol{\xi}$ to obtain $P(\xi)$. Tensor product quadrature approach [39] can be used to calculate the numerical integration in (18), which can be expressed by

$$
\int P(\boldsymbol{\xi}) \psi_{k}(\boldsymbol{\xi}) w(\boldsymbol{\xi}) d \boldsymbol{\xi} \approx \sum_{q=1}^{N} P\left(\boldsymbol{\xi}_{q}\right) \psi_{k}\left(\boldsymbol{\xi}_{q}\right) w\left(\boldsymbol{\xi}_{q}\right),
$$

where $q$ denotes the $q$ th collocation points and $N$ denotes the total number of collocation points. In this paper, the collocation points are selected from the roots of the Legendre polynomial of a one degree higher than the order $\gamma$. Then the coefficient $u_{k}$ can be calculated by the following equation:

$$
u_{k} \approx \frac{1}{\left\langle\psi_{k}^{2}\right\rangle} \sum_{q=1}^{N} P\left(\boldsymbol{\xi}_{q}\right) \psi_{k}\left(\boldsymbol{\xi}_{q}\right) w\left(\boldsymbol{\xi}_{q}\right) .
$$

We can also rewrite (20) as

$$
u_{k} \approx \sum_{q=1}^{N} P\left(\xi_{q}\right) a_{k}\left(\xi_{q}\right)
$$

where

$$
a_{k}\left(\boldsymbol{\xi}_{q}\right)=\frac{1}{\left\langle\psi_{k}^{2}\right\rangle} \psi_{k}\left(\boldsymbol{\xi}_{q}\right) w\left(\boldsymbol{\xi}_{q}\right) .
$$

Repeating (21) from $k=0$ to $k=s-1$, the coefficients vector can be determined as

$$
\begin{aligned}
{\left[u_{0}, \ldots, u_{s-1}\right] } & =\left[P\left(\xi_{1}\right), \ldots, P\left(\xi_{N}\right)\right] \mathbf{A}(\xi), \\
\mathbf{A}(\xi) & =\left[\begin{array}{ccc}
a_{0}\left(\xi_{1}\right) & \cdots & a_{s-1}\left(\xi_{1}\right) \\
\vdots & \ddots & \vdots \\
a_{0}\left(\xi_{N}\right) & \cdots & a_{s-1}\left(\xi_{N}\right)
\end{array}\right] .
\end{aligned}
$$

Once the coefficients are determined, the statistics of the frequency response $P(\xi)$ can be obtained. The mean $\mu$ and the variance $\sigma^{2}$ of the frequency response $P(\xi)$ can be calculated as follows:

$$
\begin{aligned}
\mu(P(\xi)) & =u_{0}, \\
\sigma^{2}(P(\xi)) & =\sum_{k=1}^{s-1} u_{k}^{2}\left\langle\psi_{k}^{2}\right\rangle .
\end{aligned}
$$




\section{The Response Surface Method for Interval Variables}

The polynomial chaos expansion method is applied to approximate to the frequency response $P$ with random variables using the sum of orthogonal polynomials. In this section, we consider the interval variable. Assume that the input vector $\boldsymbol{\eta}$ belongs to a bounded interval vector:

$$
\begin{aligned}
& \boldsymbol{\eta} \in \boldsymbol{\eta}^{I}=[\underline{\boldsymbol{\eta}}, \overline{\boldsymbol{\eta}}]=\left(\boldsymbol{\eta}_{l}^{I}\right), \\
& \eta_{l} \in \eta_{l}^{I}=\left[\underline{\eta_{l}}, \overline{\eta_{l}}\right],
\end{aligned}
$$

$$
l=0,2, \ldots, m-1
$$

where $\overline{\eta_{l}}$ and $\eta_{l}$ denote the upper and lower bounds of the $l$ th interval parameter $\eta_{l} . m$ denotes the total number of interval parameters. Referring to the work of Zou et al. [25], the response surface method can be employed to construct the approximate relationship between the response $Q$ and input variable $\boldsymbol{\eta}$. The construction of the response surface is most frequently performed by a least-squares fit to a linear metamodel [40], which can be given by

$$
Q \approx g(\boldsymbol{\eta})=b_{0}+\sum_{l=1}^{m} b_{1 l} \eta_{l}+\sum_{l<n} b_{l n} \eta_{l} \eta_{n}+\cdots,
$$

where $b_{0}, b_{l}, b_{l n}, \ldots$ are undetermined coefficients. For example, the first-order response surface function can be expressed by

$$
Q \approx g(\boldsymbol{\eta})=b_{0}+\sum_{l=1}^{m} b_{1 l} \eta_{l}
$$

The terms in (28) can be divided into two parts: one is the coefficient and the other is the base. If $m=2$, the bases in (28) can be shown as follows:

$$
\begin{aligned}
& f_{0}\left(\eta_{1}, \eta_{2}\right)=1, \\
& f_{1}\left(\eta_{1}, \eta_{2}\right)=\eta_{1}, \\
& f_{2}\left(\eta_{1}, \eta_{2}\right)=\eta_{2} .
\end{aligned}
$$

The second-order response surface function can be written as

$$
Q \approx g(\boldsymbol{\eta})=b_{0}+\sum_{l=1}^{m} b_{1 l} \eta_{l}+\sum_{l=1}^{m} b_{2 l} \eta_{l}^{2}+\sum_{l<n} b_{3 l} \eta_{l} \eta_{n}
$$

Assume that the total number of the terms in (30) is $t$. Then (30) can be expressed by

$$
\begin{aligned}
Q & \approx g(\boldsymbol{\eta})=\sum_{l=0}^{t-1} b_{l} \varphi_{l}=\mathbf{b}^{T} \boldsymbol{\varphi}, \\
\mathbf{b} & =\left[b_{0}, \ldots, b_{t-1}\right]^{T}, \\
\boldsymbol{\varphi} & =\left[\varphi_{0}, \ldots, \varphi_{t-1}\right]^{T} .
\end{aligned}
$$

The bases in (33) can be shown as follows:

$$
\begin{aligned}
& \varphi_{0}=f\left(\eta_{1}, \eta_{2}\right)=1, \\
& \varphi_{1}=f\left(\eta_{1}, \eta_{2}\right)=\eta_{1}, \\
& \varphi_{2}=f\left(\eta_{1}, \eta_{2}\right)=\eta_{2}, \\
& \varphi_{3}=f\left(\eta_{1}, \eta_{2}\right)=\eta_{1}^{2}, \\
& \varphi_{4}=f\left(\eta_{1}, \eta_{2}\right)=\eta_{2}^{2}, \\
& \varphi_{5}=f\left(\eta_{1}, \eta_{2}\right)=\eta_{1} \eta_{2} .
\end{aligned}
$$

The undetermined coefficient vector $\mathbf{b}$ can be calculated by the linear regression method, and the sampling points are generated by Latin hypercube design [41]. It is recommended that the selected sampling points must be more than twice the number of coefficients for obtaining robust estimates. Assuming that the number of the selected sampling points is $r$, the coefficient vector $\mathbf{b}$ can be obtained by

$$
\begin{aligned}
\mathbf{b} & =\left(\mathbf{F}(\boldsymbol{\eta})^{T} \mathbf{F}(\boldsymbol{\eta})\right)^{-1} \mathbf{F}(\boldsymbol{\eta})^{T} \mathbf{G}, \\
\mathbf{F}(\boldsymbol{\eta}) & =\left[\begin{array}{ccc}
\varphi_{0}\left(\boldsymbol{\eta}_{1}\right) & \cdots & \varphi_{0}\left(\boldsymbol{\eta}_{r}\right) \\
\vdots & \ddots & \vdots \\
\varphi_{t-1}\left(\boldsymbol{\eta}_{1}\right) & \cdots & \varphi_{t-1}\left(\boldsymbol{\eta}_{r}\right)
\end{array}\right], \\
\mathbf{G} & =\left[g\left(\boldsymbol{\eta}_{1}\right), \ldots, g\left(\boldsymbol{\eta}_{r}\right)\right]^{T},
\end{aligned}
$$

where $\mathbf{F}(\boldsymbol{\eta})$ denotes the transform matrix, $\mathbf{G}$ is the model output vector at the selected points, and the superscript " $T$ " suggests a transposition operator.

After constructing the response surface function $g(\boldsymbol{\eta})$, all existing interval analysis methods can be employed to calculate the intervals of the simulation results in acoustic field. Since $g(\boldsymbol{\eta})$ is the function with respect to the interval vector $\boldsymbol{\eta}$, the overestimation is unavoidable according to interval mathematics. MCS method is a reliable and robust alternative for calculating the range of simulation results by a combination with $g(\boldsymbol{\eta})$. The main steps of MCS method can be found in $[25,42]$.

\section{The Hybrid Method for Random and Interval Variables}

In this section, we consider that the acoustic system contains both uniform random vector $\boldsymbol{\xi}$ and interval vector $\boldsymbol{\eta}$. The PCRS method is proposed, which is designed with nonintrusiveness and effectiveness in calculating the intervals of probabilistic characteristics of response of the acoustic system with hybrid parameters. The PCRS method stems from the incorporation of polynomial chaos method and response method. Then, the upper and lower bounds of probabilistic characteristics can be calculated by means of the PCRS combined with the existing interval analysis method.

5.1. The Construction of the PCRS Method. Firstly, the interval vector $\boldsymbol{\eta}$ is regarded as a constant vector. Based on the 
polynomial chaos expansion method, the frequency response $P(\boldsymbol{\xi}, \boldsymbol{\eta})$ can be expanded by $(10)$ :

$$
P(\boldsymbol{\xi}, \boldsymbol{\eta}) \approx \sum_{k=0}^{s-1} u_{k} \psi_{k}(\boldsymbol{\xi})
$$

As $\psi_{k}(\boldsymbol{\xi})$ is the Legendre polynomials which is only the function of $\boldsymbol{\xi}, u_{k}$ will be the function of $\boldsymbol{\eta}$, namely, $u_{k}(\boldsymbol{\eta})$.

Secondly, based on the response surface method, $u_{k}(\boldsymbol{\eta})$ can be expanded using (31):

$$
u_{k}(\boldsymbol{\eta}) \approx \sum_{l=0}^{t-1} b_{k l} \varphi_{l}(\boldsymbol{\eta})
$$

Substituting (39) into (38), we can obtain the approximation of the frequency response as follows:

$$
P(\boldsymbol{\xi}, \boldsymbol{\eta}) \approx \sum_{k=0}^{s-1} \sum_{l=0}^{t-1} b_{k l} \varphi_{l}(\boldsymbol{\eta}) \psi_{k}(\boldsymbol{\xi})
$$

In order to obtain the explicit format of the frequency response $P(\boldsymbol{\xi}, \boldsymbol{\eta})$, the undetermined coefficient $b_{k l}$ in (40) is required to be solved. The coefficient vector $\mathbf{b}_{k}=\left[b_{k, 0}\right.$, $\left.\ldots, b_{k, t-1}\right]^{T}$ can be calculated by (35) as follows:

$$
\left[\begin{array}{c}
b_{k, 0} \\
\vdots \\
b_{k, t-1}
\end{array}\right]=\left(\mathbf{F}(\boldsymbol{\eta})^{T} \mathbf{F}(\boldsymbol{\eta})\right)^{-1} \mathbf{F}(\boldsymbol{\eta})^{T}\left[\begin{array}{c}
u_{k}\left(\boldsymbol{\eta}_{1}\right) \\
\vdots \\
u_{k}\left(\boldsymbol{\eta}_{r}\right)
\end{array}\right] \text {, }
$$

where $u_{k}\left(\boldsymbol{\eta}_{l}\right)$ denotes $\boldsymbol{u}_{k}(\boldsymbol{\eta})$ at the sampling points $\boldsymbol{\eta}_{l}$. Traversing $k$ from 0 to $s-1$, the coefficient vector $\mathbf{B}$ can be obtained:

$$
\begin{aligned}
\mathbf{B} & =\left[\begin{array}{ccc}
b_{0,0} & \cdots & b_{s-1,0} \\
\vdots & \ddots & \vdots \\
b_{0, t-1} & \cdots & b_{s-1, t-1}
\end{array}\right] \\
& =\left(\mathbf{F}(\boldsymbol{\eta})^{T} \mathbf{F}(\boldsymbol{\eta})\right)^{-1} \mathbf{F}(\boldsymbol{\eta})^{T}\left[\begin{array}{ccc}
u_{0}\left(\boldsymbol{\eta}_{1}\right) & \cdots & u_{s-1}\left(\boldsymbol{\eta}_{1}\right) \\
\vdots & \ddots & \vdots \\
u_{0}\left(\boldsymbol{\eta}_{r}\right) & \cdots & u_{s-1}\left(\boldsymbol{\eta}_{r}\right)
\end{array}\right] .
\end{aligned}
$$

Then using (23), the function value vector $\left[u_{0}\left(\boldsymbol{\eta}_{i}\right), \ldots\right.$, $\left.u_{s-1}\left(\boldsymbol{\eta}_{i}\right)\right]$ can be calculated as

$$
\begin{aligned}
& {\left[u_{0}\left(\boldsymbol{\eta}_{i}\right), \ldots, u_{s-1}\left(\boldsymbol{\eta}_{i}\right)\right]} \\
& \quad=\left[P\left(\boldsymbol{\xi}_{1}, \boldsymbol{\eta}_{i}\right), \ldots, P\left(\boldsymbol{\xi}_{N}, \boldsymbol{\eta}_{i}\right)\right] \mathbf{A}(\boldsymbol{\xi}),
\end{aligned}
$$

where $\boldsymbol{\eta}_{i}$ denotes the $i$ th of the sampling points vector $\boldsymbol{\eta}$. Repeating (43) from $i=0$ to $i=r$, the function value vector in the right side of (42) can be expressed as

$$
\begin{aligned}
& {\left[\begin{array}{ccc}
u_{0}\left(\boldsymbol{\eta}_{1}\right) & \cdots & u_{s-1}\left(\boldsymbol{\eta}_{1}\right) \\
\vdots & \ddots & \vdots \\
u_{0}\left(\boldsymbol{\eta}_{r}\right) & \cdots & u_{s-1}\left(\boldsymbol{\eta}_{r}\right)
\end{array}\right]} \\
& \quad=\left[\begin{array}{ccc}
P\left(\boldsymbol{\xi}_{1}, \boldsymbol{\eta}_{1}\right) & \cdots & P\left(\boldsymbol{\xi}_{N}, \boldsymbol{\eta}_{1}\right) \\
\vdots & \ddots & \vdots \\
P\left(\boldsymbol{\xi}_{1}, \boldsymbol{\eta}_{r}\right) & \cdots & P\left(\boldsymbol{\xi}_{N}, \boldsymbol{\eta}_{r}\right)
\end{array}\right] \mathbf{A}(\boldsymbol{\xi}) .
\end{aligned}
$$

Substituting (44) into (42), the coefficient vector B can be rewritten as

$$
\begin{aligned}
& \mathbf{B}=\left(\mathbf{F}(\boldsymbol{\eta})^{T} \mathbf{F}(\boldsymbol{\eta})\right)^{-1} \mathbf{F}(\boldsymbol{\eta})^{T} \mathbf{P A}(\boldsymbol{\xi}), \\
& \mathbf{P}=\left[\begin{array}{ccc}
P\left(\boldsymbol{\xi}_{1}, \boldsymbol{\eta}_{1}\right) & \cdots & P\left(\boldsymbol{\xi}_{N}, \boldsymbol{\eta}_{1}\right) \\
\vdots & \ddots & \vdots \\
P\left(\boldsymbol{\xi}_{1}, \boldsymbol{\eta}_{r}\right) & \cdots & P\left(\boldsymbol{\xi}_{N}, \boldsymbol{\eta}_{r}\right)
\end{array}\right],
\end{aligned}
$$

where $\mathbf{P}$ is the frequency response value matrix of original system at the collocation points and sampling points. As mentioned above in Sections 3 and 4, the total number of collocation points is $N$ and the collocation points are selected from the roots of the Legendre polynomial of a one degree higher than the order $\gamma$. And the number of the selected sampling points that is $r$ must be more than twice the number of coefficients $t$ for obtaining robust estimates. Once the collocation points and sampling points are selected, the frequency response value matrix of $\mathbf{P}$ can be obtained by calculating (8) $N \times r$ times.

By substituting the frequency response value matrix of $\mathbf{P}$ in (46) into (45), the coefficient vector B can be obtained. Then the original model in (8) can be approximated by (40), which can be written as follows:

$$
\mathbf{P}(\boldsymbol{\xi}, \boldsymbol{\eta})=\mathbf{Z}^{-1}(\boldsymbol{\xi}, \boldsymbol{\eta}) \mathbf{q}(\boldsymbol{\xi}, \boldsymbol{\eta}) \approx \sum_{k=0}^{s-1} \sum_{l=0}^{t-1} b_{k l} \varphi_{l}(\boldsymbol{\eta}) \psi_{k}(\boldsymbol{\xi})
$$

5.2. The Bounds of the Statistical Characteristics of the Frequency Response. After constructing the PCRS model via (47), the mean and variance of the frequency response need to be evaluated. Substituting (39) into (25), the mean of the frequency response $P(\boldsymbol{\xi}, \boldsymbol{\eta})$ can be written as

$$
\mu(P(\boldsymbol{\xi}, \boldsymbol{\eta}))=u_{0}(\boldsymbol{\eta})=\sum_{l=0}^{t-1} b_{0, l} \varphi_{l}(\boldsymbol{\eta})
$$


TABLE 1: Characteristics of the uncertain parameters of the 2D rectangular cavity.

\begin{tabular}{lcccc}
\hline Parameters & $c(\mathrm{~m} / \mathrm{s})$ & $\rho\left(\mathrm{kg} / \mathrm{m}^{3}\right)$ & $A_{1}(\mathrm{~m} / \mathrm{s} / \mathrm{Pa})$ & $A_{2}(\mathrm{~m} / \mathrm{s} / \mathrm{Pa})$ \\
\hline Values & $U(329.8,350.2)$ & $U(1.1495,1.2705)$ & {$[0.0009,0.0011]$} & {$[0.0018,0.0022]$} \\
\hline
\end{tabular}

Similarly, the variance of the frequency response $P(\boldsymbol{\xi}, \boldsymbol{\eta})$ can be expressed by

$$
\begin{aligned}
\sigma^{2}(P(\boldsymbol{\xi}, \boldsymbol{\eta})) & =\sum_{k=1}^{s-1} u_{k}^{2}(\boldsymbol{\eta})\left\langle\psi_{k}^{2}\right\rangle \\
& =\sum_{k=1}^{s-1}\left(\sum_{l=0}^{t-1} b_{k l} \varphi_{l}(\boldsymbol{\eta})\right)^{2}\left\langle\psi_{k}^{2}\right\rangle .
\end{aligned}
$$

It is obvious that the mean and variance of the frequency response also contain interval variables. The direct implementation of interval arithmetic is difficult to obtain reliable estimates of the mean and variance, as interval overestimation occurs due to the multioccurrence of the interval variables. In interval analysis, when the evaluated functions are multimodal, the MCS or global optimization algorithms are often employed to obtain the bounds of the functions with interval variables. If the dimension of the interval variable is less than 4, MCS can be a robust and convenient method to determine the upper and lower bounds of the functions. When the problem is high-dimensional, some global optimization algorithms such as the particle swarm algorithm and the genetic algorithms are effective to calculate the minimum and maximum values of the multimodal functions $[27,43]$. Then (48) and (49) can be expressed in the following optimization forms:

$$
\begin{aligned}
& \mu(P(\boldsymbol{\xi}, \boldsymbol{\eta}))^{I}=[\underline{\mu}(P(\boldsymbol{\xi}, \boldsymbol{\eta})), \bar{\mu}(P(\boldsymbol{\xi}, \boldsymbol{\eta}))] \\
&= {\left[\min _{-1 \leq \boldsymbol{\eta} \leq 1} \sum_{l=0}^{t-1} b_{0, l} \varphi_{l}(\boldsymbol{\eta}), \max _{-1 \leq \boldsymbol{\eta} \leq 1} \sum_{l=0}^{t-1} b_{0, l} \varphi_{l}(\boldsymbol{\eta})\right], } \\
& \sigma^{2}(P(\boldsymbol{\xi}, \boldsymbol{\eta}))^{I}=\left[\underline{\sigma}^{2}(P(\boldsymbol{\xi}, \boldsymbol{\eta})), \bar{\sigma}^{2}(P(\boldsymbol{\xi}, \boldsymbol{\eta}))\right] \\
&=\left[\min _{-1 \leq \boldsymbol{\eta} \leq 1} \sum_{k=1}^{s-1}\left(\sum_{l=0}^{t-1} b_{k l} \varphi_{l}(\boldsymbol{\eta})\right)^{2}\left\langle\psi_{k}^{2}\right\rangle,\right. \\
&\left.\max _{-1 \leq \boldsymbol{\eta} \leq 1} \sum_{k=1}^{s-1}\left(\sum_{l=0}^{t-1} b_{k l} \varphi_{l}(\boldsymbol{\eta})\right)^{2}\left\langle\psi_{k}^{2}\right\rangle\right] .
\end{aligned}
$$

As aforementioned, there are mainly four steps to determine the bounds of probabilistic features of the frequency response: (1) produce the collocation points for random variables and the sampling points for interval variables according to the order of the Legendre polynomials and the number of undetermined coefficients of the response surface function, respectively; (2) run the original acoustic model at each points vector to obtain the values of the frequency response value matrix $\mathbf{P}$; (3) build the coefficient vector $\mathbf{B}$ and then obtain the final expression of PCRS model of the frequency response $P(\boldsymbol{\xi}, \boldsymbol{\eta})$; (4) calculate the bounds of probabilistic

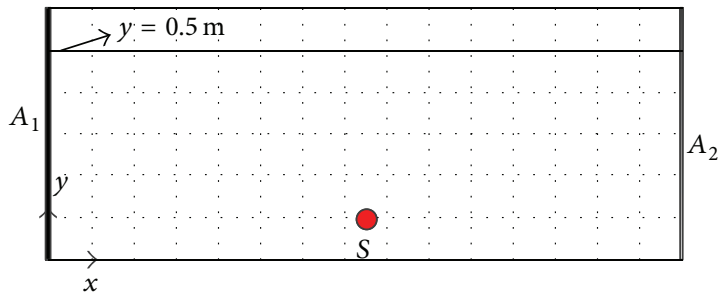

FIgURE 1: Finite element mesh of the 2D rectangular cavity.

features of the frequency response based on the PCRS model using the interval analysis method.

\section{Numerical Study}

6.1. Numerical Study of a 2D Rectangular Cavity. To demonstrate the effectiveness of the proposed PCRS method, a 2D rectangular cavity is studied in this section. Figure 1 depicts a $2 \mathrm{D}$ cavity model of dimensions $1.5 \times 0.6 \mathrm{~m}$. The acoustic cavity is discretized by the 8 -node quadrilateral elements. The mesh counts $15 \times 6$ elements with a total of 313 nodes. The left-hand side and the right-hand side of the cavity are covered with absorbing material, named $A_{1}$ and $A_{2}$, respectively. The fluid is excited by a unit point sound source located at Node 197 $(0.75 \mathrm{~m}$ and $0.3 \mathrm{~m})$. Considering the unpredictable environment temperature, the density of air and the acoustic speed of air surrounding the acoustic cavity are assumed as random variables which follow the uniform distribution. Because of the manufacturing errors and the measured errors, the admittance coefficients of the two absorbing materials are regarded as interval variables. These uncertain parameters are assumed to be independent of each other. The values of the uncertain parameters are shown in Table 1.

The lower and upper bounds of expectation and variance of the frequency response amplitude at nodes along the line of $y=0.5 \mathrm{~m}$ are calculated by the PCRS method. In this case, we choose the order of polynomial chaos as 2 and the order of response surface as 2 . The number of the collocation points for random parameters is $9(N=9)$, and the sampling points is $50(r=50)$. The total number of samples is $N \times$ $r=9 \times 50=450$, which is used to obtain the frequency response value matrix of original system. After obtaining the coefficient vector $\mathbf{B}$, the intervals of the expectation and variance of the frequency response can be evaluated by (50).

The results obtained by the direct MCS method based on the original numerical model are used as the reference solutions for validating the accuracy of the proposed method. It should be noted that in the direct MCS method the number of sampling points for interval parameters is 1000 and the size of sampling points for random parameters is $10^{4}$. In other words, the total number of the samples is $1000 \times 10^{4}$. 
TABLE 2: Execution time of the MCS method and the PCRS method.

\begin{tabular}{|c|c|c|c|}
\hline \multicolumn{2}{|c|}{ MCS } & \multicolumn{2}{|c|}{ PCRS } \\
\hline $\begin{array}{l}\text { Total } \\
\text { execution } \\
\text { time (s) }\end{array}$ & $\begin{array}{l}\text { The execution } \\
\text { time of } \\
\text { obtaining the } \\
\text { values of } \mathbf{P}(\mathrm{s})\end{array}$ & $\begin{array}{l}\text { The execution } \\
\text { time of } \\
\text { calculating the } \\
\text { values of } \mathbf{B}(\mathrm{s})\end{array}$ & $\begin{array}{l}\text { Total } \\
\text { execution time } \\
\text { (s) }\end{array}$ \\
\hline 322403.30 & 27.75 & 0.06 & 41.25 \\
\hline
\end{tabular}

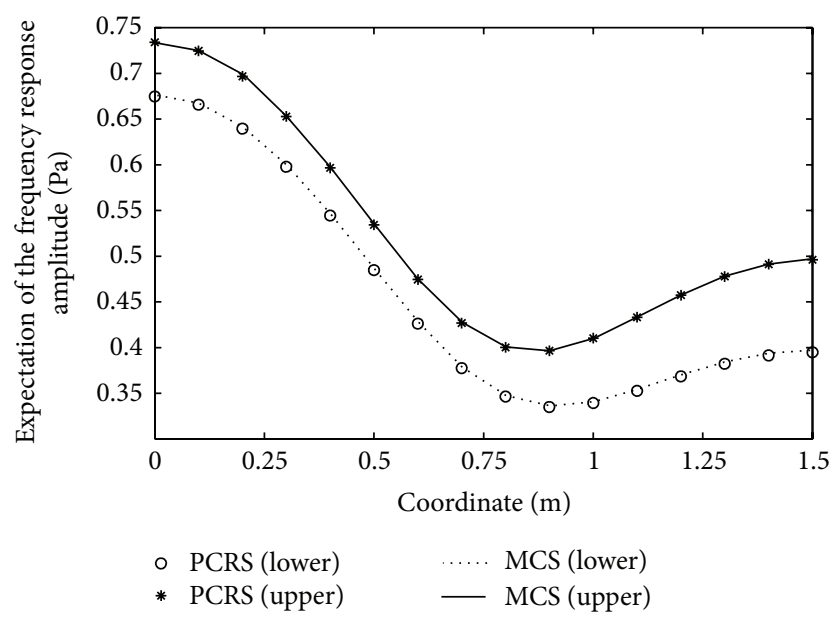

Figure 2: The lower and upper bounds of expectation of the frequency response amplitude at nodes along the line of $y=0.5 \mathrm{~m}$ calculated by PCRS and MCS.

Compared with the direct MCS method, the PCRS method is more efficient, as it only computes the original frequency response value 300 times. As shown in Table 2, we can see that the total execution time of the MCS method is much longer than that of the PCRS method. Furthermore, it takes about $27.75 \mathrm{~s}$ to obtain the values of the frequency response matrix $\mathbf{P}$ in the PCRS method. And it only takes 0.06 s to obtain coefficient vector $\mathbf{B}$, which means that it takes little time for constructing the PCRS model after obtaining the frequency value matrix $\mathbf{P}$.

The lower and upper bounds of expectation and variance of the frequency response at nodes along the line of $y=0.5 \mathrm{~m}$ are shown in Figures 2 and 3 for frequency $100 \mathrm{~Hz}$. The results show that the intervals of expectation and variance obtained by PCRS match the lower and upper bounds yielded by MCS perfectly. So the PCRS can provide sufficient accuracy to evaluate the interval expectation and variance of the frequency response. To investigate the accuracy of PCRS for predicting the acoustic frequency response more clearly, the intervals of expectation and variance and the relative errors are listed in Tables 3 and 4. The results show that the relative errors of the proposed method are acceptable compared with the MCS solutions. Furthermore, the relative errors of the bounds of variances are larger than those of the bounds of expectation, which means that the bounds of the variance are more sensitive to the uncertain parameters compared to the bounds of the expectation.

Based on (47), the MCS method with $5 \times 10^{3}$ sampling points for interval parameters and $10^{3}$ collocation points for

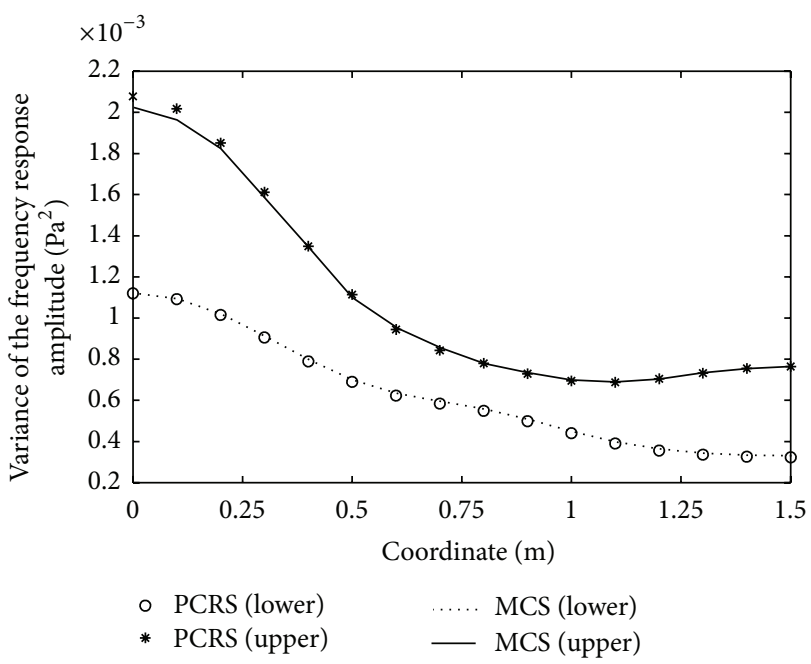

FIGURE 3: The lower and upper bounds of variance of the frequency response amplitude at nodes along the line of $y=0.5 \mathrm{~m}$ calculated by PCRS and MCS.

random parameters can be used to compute the intervals of PDF of the response. The direct MCS with total $5 \times 10^{6}$ samples based on the original numerical model is used as the referenced solution. The lower and upper bounds of the response PDF at node $R 1(x=0.6, y=0.5)$ and node $R 2(x=$ $1.4, y=0.5)$ using the direct MCS and the PCRS are plotted in Figures 4 and 5. It can be seen that the overall shapes of the probability distribution mapped by the PCRS method are close to the direct MCS results, which again verify the effectiveness of the proposed method.

The effects of the uncertain levels of the interval variables on the accuracy of the PCRS method are investigated. The considered uncertain levels of admittance coefficients of the two absorbing materials are $5 \%, 10 \%, 15 \%$, and $20 \%$, respectively. Because the variance is more sensitive to uncertain levels of interval variables than the expectation, we only research the bounds of variance of the frequency response amplitude of the nodes along the line of $y=0.5 \mathrm{~m}$ at different uncertainty levels of the interval variables. The considered frequency is $200 \mathrm{~Hz}$. It can be seen from Figures 6-9 that the intervals of variance of the frequency response obtained by the PCRS method match the intervals calculated by the MCS method well until the uncertain level of the interval variables reaches $15 \%$. It indicates that the proposed method has good accuracy to predict the intervals of expectation and variance of the frequency response of the acoustic system with both random and interval variables. We can also find that the larger the uncertain level of the interval variables is, the larger the deviation is between the intervals calculated by the PCRS and that obtained by the MCS method.

6.2. Numerical Study of a 2D Sedan Passenger Compartment. In this section, a case of study of a 2D car cavity is adopted to investigate the performance of PCRS. This cavity is similar to the one used in the earlier studies by other authors [44, 45]. 
TABLE 3: The relative errors of the lower and upper bounds of expectation of the frequency response amplitude along the line of $y=0.5 \mathrm{~m}$.

\begin{tabular}{|c|c|c|c|c|c|c|}
\hline \multirow{2}{*}{ Coordinate (m) } & \multicolumn{3}{|c|}{ Lower bounds } & \multicolumn{3}{|c|}{ Upper bounds } \\
\hline & Monte Carlo & PCRS & Relative errors (\%) & Monte Carlo & PCRS & Relative errors (\%) \\
\hline 0 & $6.77 E-01$ & $6.75 E-01$ & $0.39 \%$ & $7.36 E-01$ & $7.34 E-01$ & $0.22 \%$ \\
\hline 0.1 & $6.68 E-01$ & $6.66 E-01$ & $0.39 \%$ & $7.26 E-01$ & $7.25 E-01$ & $0.22 \%$ \\
\hline 0.2 & $6.42 E-01$ & $6.39 E-01$ & $0.39 \%$ & $6.98 E-01$ & $6.97 E-01$ & $0.22 \%$ \\
\hline 0.3 & $6.00 E-01$ & $5.98 E-01$ & $0.40 \%$ & $6.54 E-01$ & $6.53 E-01$ & $0.23 \%$ \\
\hline 0.4 & $5.47 E-01$ & $5.44 E-01$ & $0.41 \%$ & $5.98 E-01$ & $5.97 E-01$ & $0.23 \%$ \\
\hline 0.5 & $4.87 E-01$ & $4.85 E-01$ & $0.43 \%$ & $5.36 E-01$ & $5.34 E-01$ & $0.24 \%$ \\
\hline 0.6 & $4.28 E-01$ & $4.26 E-01$ & $0.46 \%$ & $4.76 E-01$ & $4.74 E-01$ & $0.26 \%$ \\
\hline 0.7 & $3.80 E-01$ & $3.78 E-01$ & $0.52 \%$ & $4.28 E-01$ & $4.27 E-01$ & $0.29 \%$ \\
\hline 0.8 & $3.48 E-01$ & $3.46 E-01$ & $0.60 \%$ & $4.01 E-01$ & $4.00 E-01$ & $0.33 \%$ \\
\hline 0.9 & $3.37 E-01$ & $3.35 E-01$ & $0.68 \%$ & $3.98 E-01$ & $3.96 E-01$ & $0.36 \%$ \\
\hline 1 & $3.42 E-01$ & $3.39 E-01$ & $0.75 \%$ & $4.12 E-01$ & $4.10 E-01$ & $0.39 \%$ \\
\hline 1.1 & $3.56 E-01$ & $3.53 E-01$ & $0.80 \%$ & $4.35 E-01$ & $4.33 E-01$ & $0.40 \%$ \\
\hline 1.2 & $3.71 E-01$ & $3.68 E-01$ & $0.83 \%$ & $4.59 E-01$ & $4.57 E-01$ & $0.41 \%$ \\
\hline 1.3 & $3.86 E-01$ & $3.82 E-01$ & $0.85 \%$ & $4.80 E-01$ & $4.78 E-01$ & $0.42 \%$ \\
\hline 1.4 & $3.95 E-01$ & $3.92 E-01$ & $0.87 \%$ & $4.93 E-01$ & $4.91 E-01$ & $0.43 \%$ \\
\hline 1.5 & $3.98 E-01$ & $3.95 E-01$ & $0.87 \%$ & $4.98 E-01$ & $4.96 E-01$ & $0.43 \%$ \\
\hline
\end{tabular}

TABLE 4: The relative errors of the lower and upper bounds of variance of the frequency response amplitude along the line of $y=0.5 \mathrm{~m}$.

\begin{tabular}{|c|c|c|c|c|c|c|}
\hline \multirow{2}{*}{ Coordinate $(\mathrm{m})$} & \multicolumn{3}{|c|}{ Lower bounds } & \multicolumn{3}{|c|}{ Upper bounds } \\
\hline & Monte Carlo & PCRS & Relative errors (\%) & Monte Carlo & PCRS & Relative errors (\%) \\
\hline 0 & $1.13 E-03$ & $1.12 E-03$ & $0.54 \%$ & $2.03 E-03$ & $2.08 E-03$ & $2.22 \%$ \\
\hline 0.1 & $1.10 E-03$ & $1.09 E-03$ & $0.60 \%$ & $1.98 E-03$ & $2.02 E-03$ & $2.16 \%$ \\
\hline 0.2 & $1.02 E-03$ & $1.01 E-03$ & $0.79 \%$ & $1.82 E-03$ & $1.85 E-03$ & $1.97 \%$ \\
\hline 0.3 & $9.16 E-04$ & $9.05 E-04$ & $1.11 \%$ & $1.59 E-03$ & $1.61 E-03$ & $1.62 \%$ \\
\hline 0.4 & $8.01 E-04$ & $7.89 E-04$ & $1.54 \%$ & $1.33 E-03$ & $1.35 E-03$ & $1.07 \%$ \\
\hline 0.5 & $7.04 E-04$ & $6.90 E-04$ & $2.00 \%$ & $1.11 E-03$ & $1.11 E-03$ & $0.34 \%$ \\
\hline 0.6 & $6.38 E-04$ & $6.23 E-04$ & $2.36 \%$ & $9.49 E-04$ & $9.44 E-04$ & $0.45 \%$ \\
\hline 0.7 & $5.99 E-04$ & $5.84 E-04$ & $2.48 \%$ & $8.52 E-04$ & $8.43 E-04$ & $1.07 \%$ \\
\hline 0.8 & $5.62 E-04$ & $5.49 E-04$ & $2.29 \%$ & $7.91 E-04$ & $7.80 E-04$ & $1.37 \%$ \\
\hline 0.9 & $5.12 E-04$ & $4.99 E-04$ & $2.52 \%$ & $7.39 E-04$ & $7.29 E-04$ & $1.37 \%$ \\
\hline 1 & $4.53 E-04$ & $4.40 E-04$ & $2.71 \%$ & $7.03 E-04$ & $6.95 E-04$ & $1.18 \%$ \\
\hline 1.1 & $4.02 E-04$ & $3.90 E-04$ & $2.91 \%$ & $6.94 E-04$ & $6.88 E-04$ & $0.88 \%$ \\
\hline 1.2 & $3.67 E-04$ & $3.56 E-04$ & $3.14 \%$ & $7.09 E-04$ & $7.05 E-04$ & $0.56 \%$ \\
\hline 1.3 & $3.47 E-04$ & $3.36 E-04$ & $3.33 \%$ & $7.35 E-04$ & $7.32 E-04$ & $0.32 \%$ \\
\hline 1.4 & $3.38 E-04$ & $3.26 E-04$ & $3.46 \%$ & $7.57 E-04$ & $7.56 E-04$ & $0.17 \%$ \\
\hline 1.5 & $3.35 E-04$ & $3.23 E-04$ & $3.50 \%$ & $7.66 E-04$ & $7.65 E-04$ & $0.12 \%$ \\
\hline
\end{tabular}

The finite element mesh of the cavity and boundary conditions are shown in Figure 10. The finite element model of the acoustic cavity consists of 152 eight-node quadrilateral acoustic elements with a total of 533 nodes. A unit point sound source excitation is placed at Node $432(0.9123 \mathrm{~m},-0.4905 \mathrm{~m})$ and the pressure responses of Node $434(1.3975 \mathrm{~m}, 0.15522 \mathrm{~m})$ and Node $519(0.68246 \mathrm{~m},-0.48195 \mathrm{~m})$ are assumed to be measured. Three boundaries are covered with three different absorbers. Due to the unpredictability of environment temperature, the air density and the sound speed are assumed as random variables which follow the uniform distribution. Because of manufacturing errors and measurement errors, the admittance coefficients of the three different absorbers are considered as the interval parameters. These parameters are assumed to be independent of each other. The random parameters and interval parameters are listed in Table 5.

The proposed method is adopted to calculate the acoustic frequency response of the sedan passenger compartment. In the implementation of the direct MCS method, the number of sampling points for interval parameters is chosen as $5 \times 10^{3}$, and the size of collocation points for random parameters is chosen as $10^{3}$. Figures 11-14 depict the lower and upper bounds of expectation and variance of the frequency response amplitude at nodes $R 1$ and $R 2$ in the frequencies ranging from 


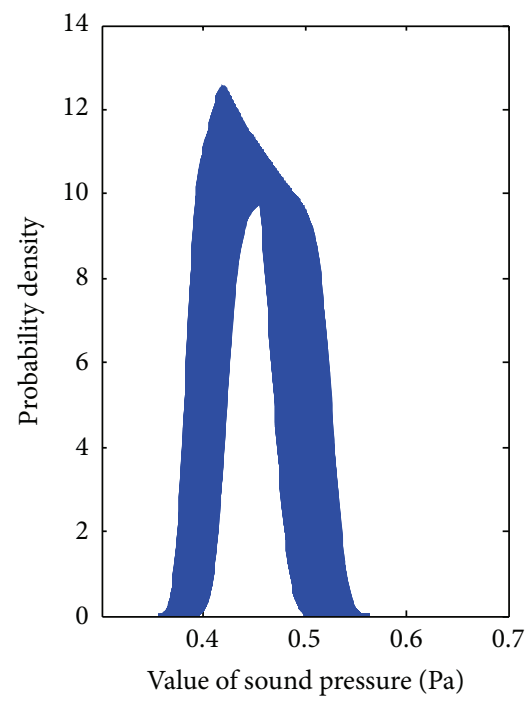

(a)

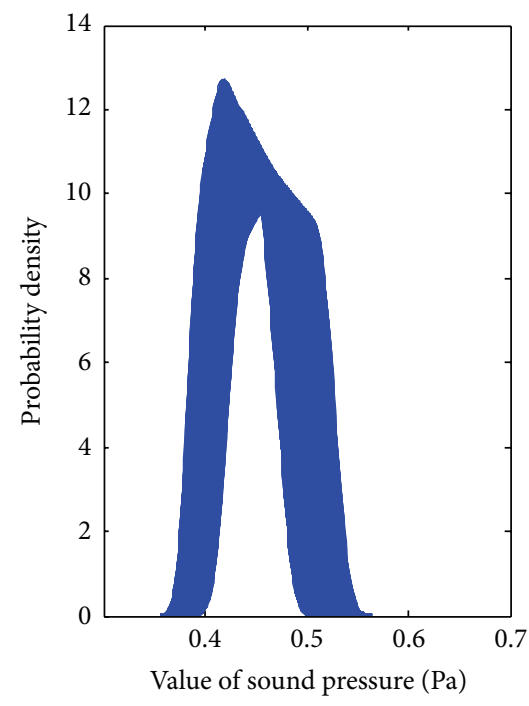

(b)

FIGURE 4: The lower and upper bounds of the response probability density of node $R 1$ calculated by (a) MCS and (b) PCRS.

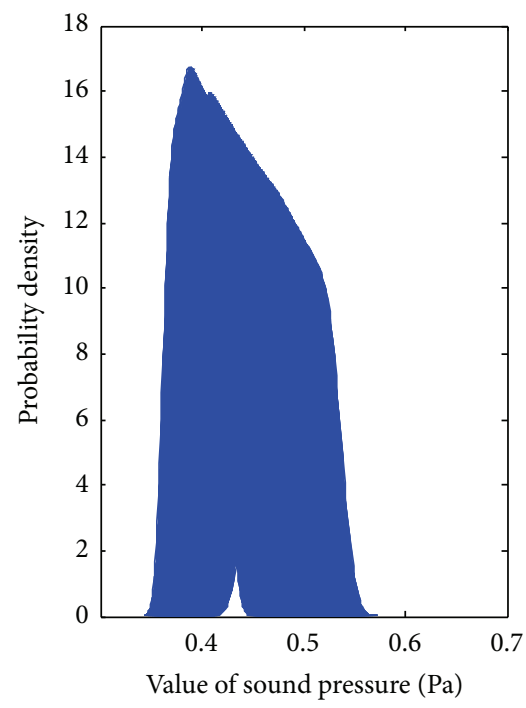

(a)

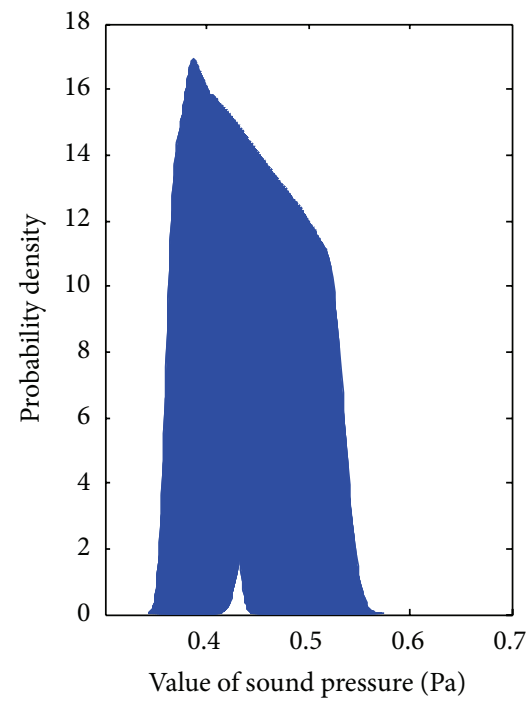

(b)

FIgURE 5: The lower and upper bounds of the response probability density of node $R 2$ calculated by (a) MCS and (b) PCRS.

$10 \mathrm{~Hz}$ to $200 \mathrm{~Hz}$. It can be seen that the results obtained by the proposed method are close to the results yielded by the MCS method. Neglecting the variation of admittance coefficients of the absorbers, when the ranges of sound speed $c$ and air density $\rho$ are assumed to be those listed in Table 5, the eigenfrequencies in the frequency band $f=5-200 \mathrm{~Hz}$ are located in frequency ranges $f=70.84-75.22 \mathrm{~Hz}$, $f=100.94-107.18 \mathrm{~Hz}, f=124.00-131.66 \mathrm{~Hz}, f=$ $150.63-159.94 \mathrm{~Hz}$, and $f=172.33-182.99 \mathrm{~Hz}$, respectively. From Figures 11 and 12, it can be found that the intervals of expectation of the frequency response are insensitive to the uncertainties of the absorbing material coefficients in the frequency band except the frequencies around the eigenfrequencies. It can be seen from Figures 13 and 14 that the uncertainties of the absorbing material coefficients have significant effect on the intervals of variance of the frequency response around the eigenfrequencies. It indicates that a small variance of the absorbing material coefficients may result in a tremendous change in the acoustic frequency response around the eigenfrequencies, which will produce large variance. Besides, the intervals of variance of the frequency response are more sensitive to the uncertainties of the absorbing material coefficients than the intervals of expectation of the frequency response. 
TABLE 5: Characteristics of the uncertain parameters of the 2D car cavity.

\begin{tabular}{lccccc}
\hline Parameters & $c(\mathrm{~m} / \mathrm{s})$ & $\rho\left(\mathrm{kg} / \mathrm{m}^{3}\right)$ & $A_{1}(\mathrm{~m} / \mathrm{s} / \mathrm{Pa})$ & $A_{2}(\mathrm{~m} / \mathrm{s} / \mathrm{Pa})$ & $A_{3}(\mathrm{~m} / \mathrm{s} / \mathrm{Pa})$ \\
\hline Values & $U(329.8,350.2)$ & $U(1.1495,1.2705)$ & {$[0.00186,0.00214]$} & {$[0.00279,0.00321]$} & {$[0.00372,0.00428]$} \\
\hline
\end{tabular}

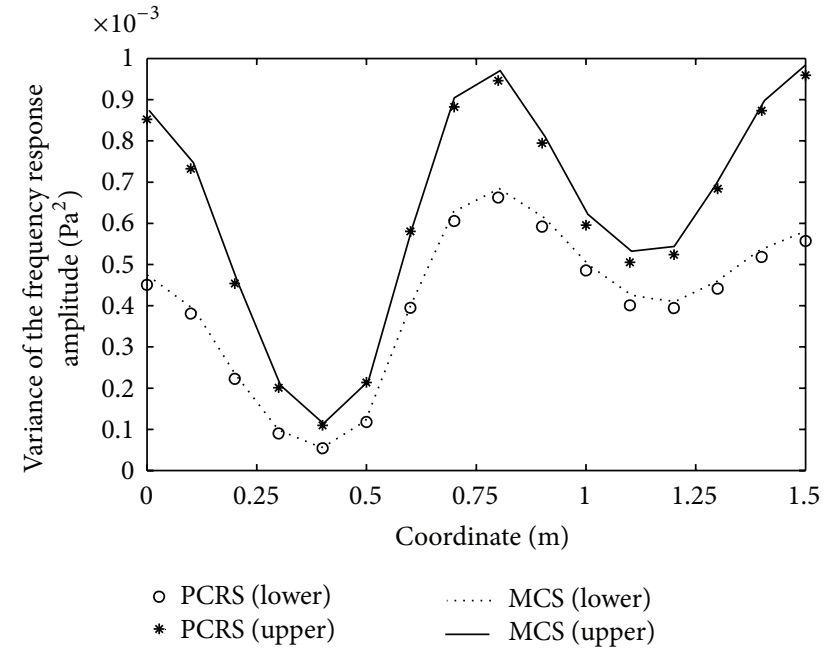

Figure 6: The lower and upper bounds of variance of the frequency response amplitude at uncertain level 5\%.

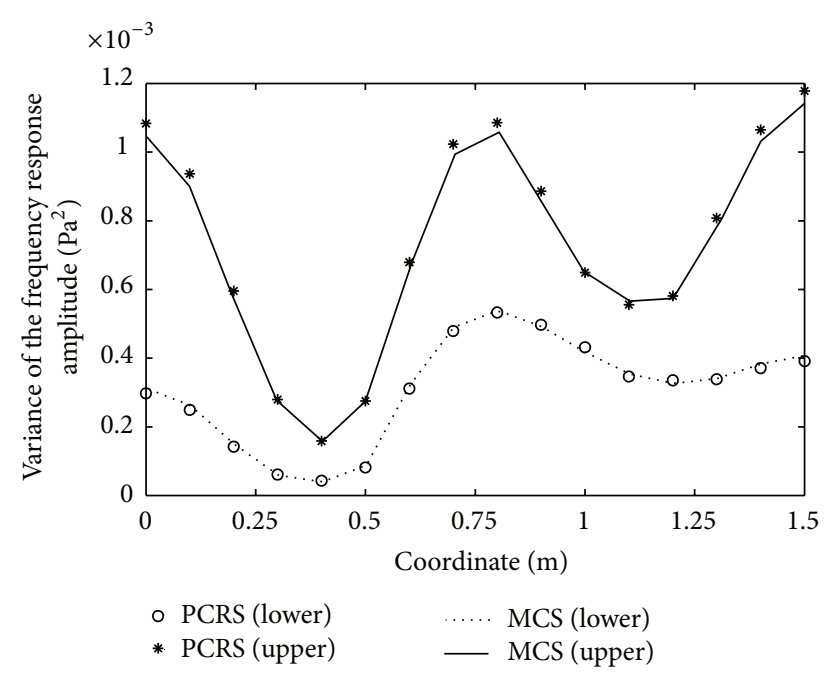

FIGURE 7: The lower and upper bounds of variance of the frequency response amplitude at uncertain level 10\%.

Based on (47), the MCS method with $5 \times 10^{3}$ sampling points for interval parameters and $10^{3}$ collocation points for random parameters can be used to compute the intervals of PDF of the response. The direct MCS with total $5 \times 10^{6}$ samples based on the original numerical model is used as the referenced solution. The lower and upper bounds of the response PDF at node $R 1$ and node $R 2$ using the direct MCS and the PCRS are plotted in Figures 15 and 16. It can be seen that the overall shapes of the probability distribution mapped by the PCRS method match well with the direct MCS

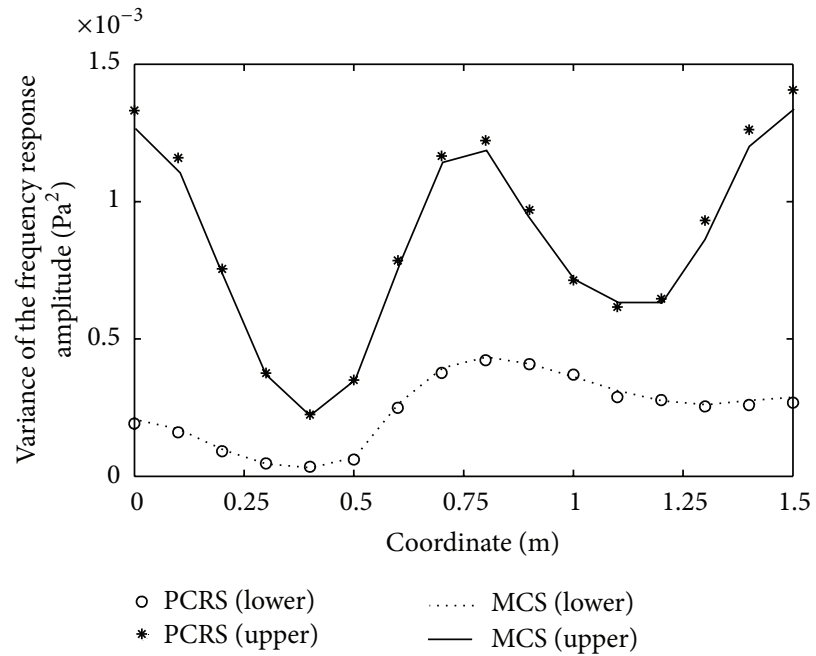

FIGURE 8: The lower and upper bounds of variance of the frequency response amplitude at uncertain level 15\%.

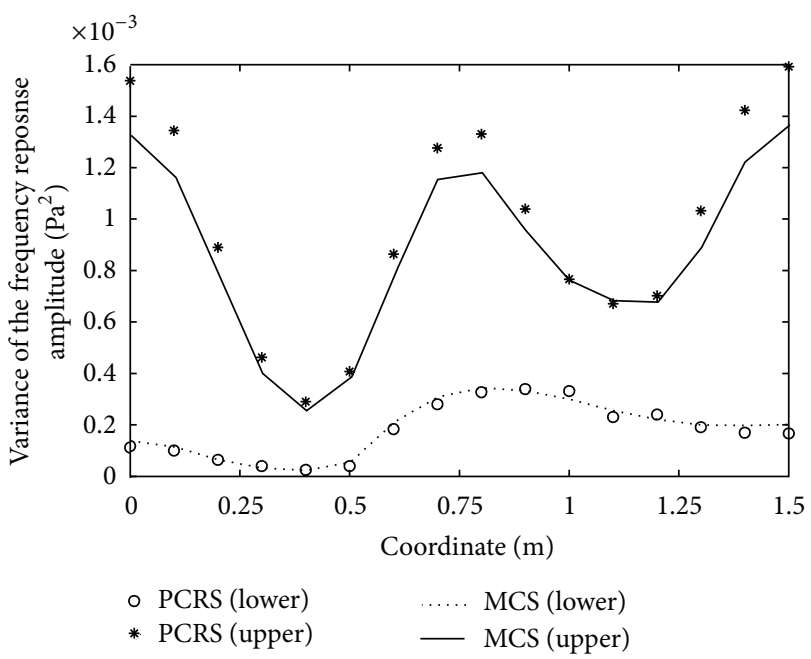

FIGURE 9: The lower and upper bounds of variance of the frequency response amplitude at uncertain level $20 \%$.

results, and the intervals of the PDF obtained by the PCRS have reasonable accuracy compared to the direct MCS results.

\section{Conclusion}

The aim of the current study is to propose a new uncertain method for uncertain propagation in acoustic system with hybrid parameters. In the proposed method, the polynomial chaos expansion method is employed to treat the random parameters, and the response surface method is used to deal with the interval parameters. Based on the PCRS model, 


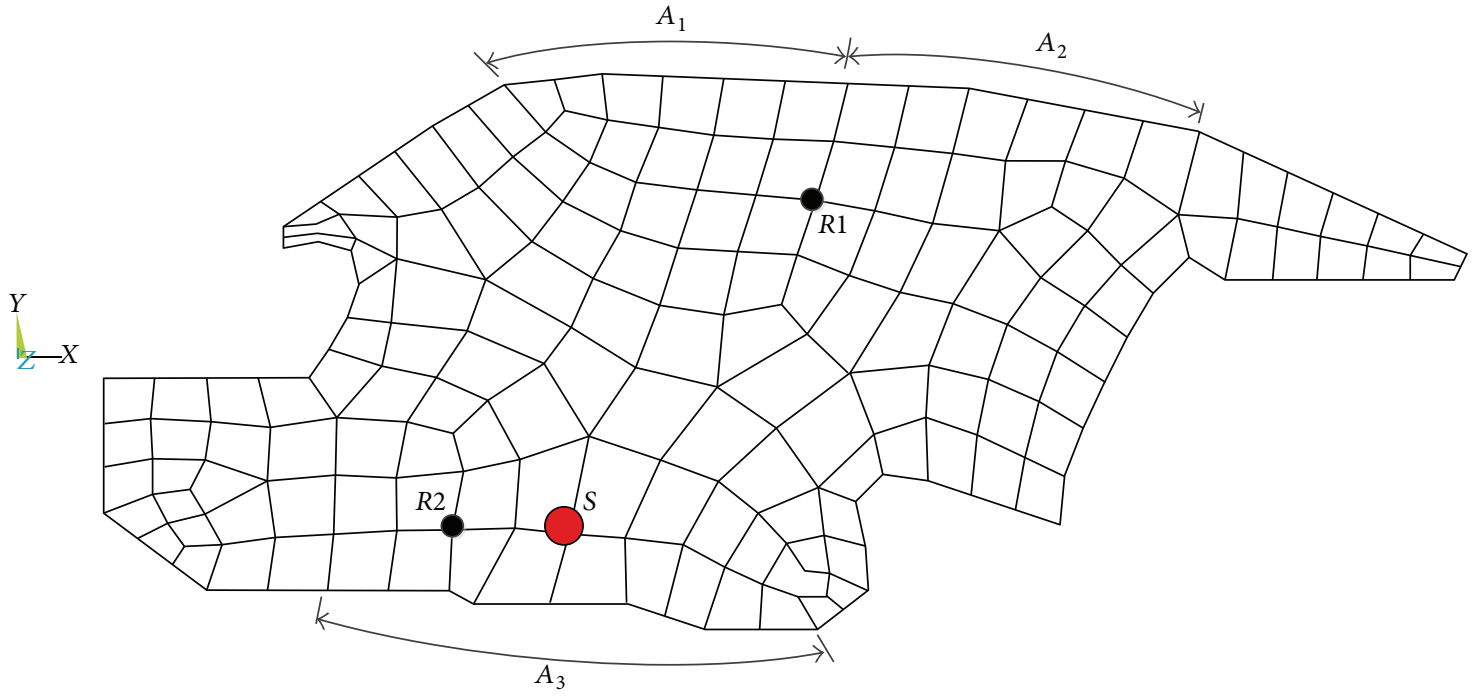

FIGURE 10: Finite element mesh of the 2D car cavity.

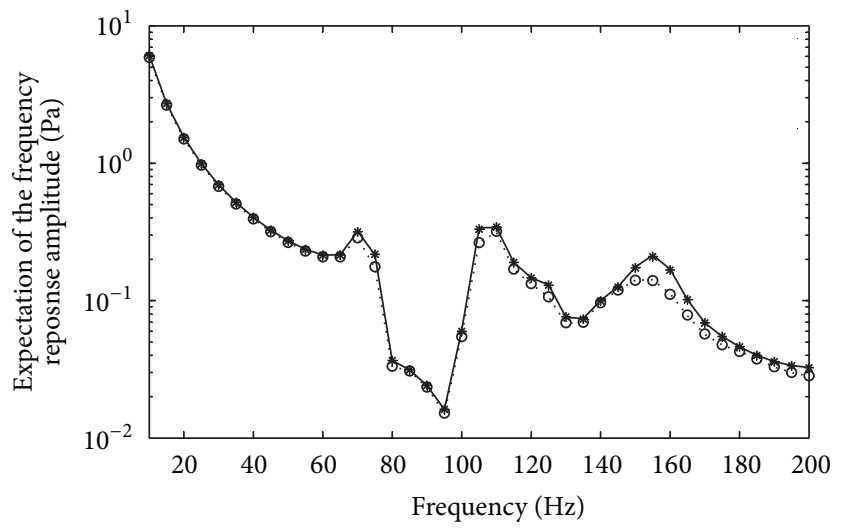

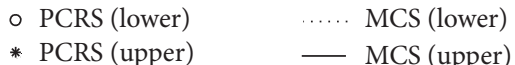

FIGURE 11: The lower and upper bounds of expectation of the frequency response amplitude at node R1 calculated by PCRS and MCS in the frequency band $f=10 \sim 200 \mathrm{~Hz}$.

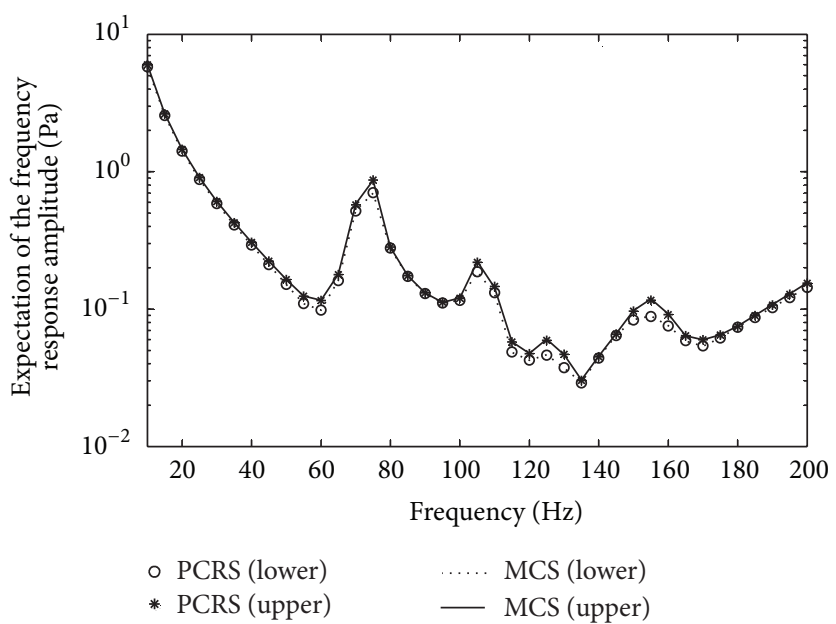

FIGURE 12: The lower and upper bounds of expectation of the frequency response amplitude at node $R 2$ calculated by PCRS and MCS in the frequency band $f=10 \sim 200 \mathrm{~Hz}$. 


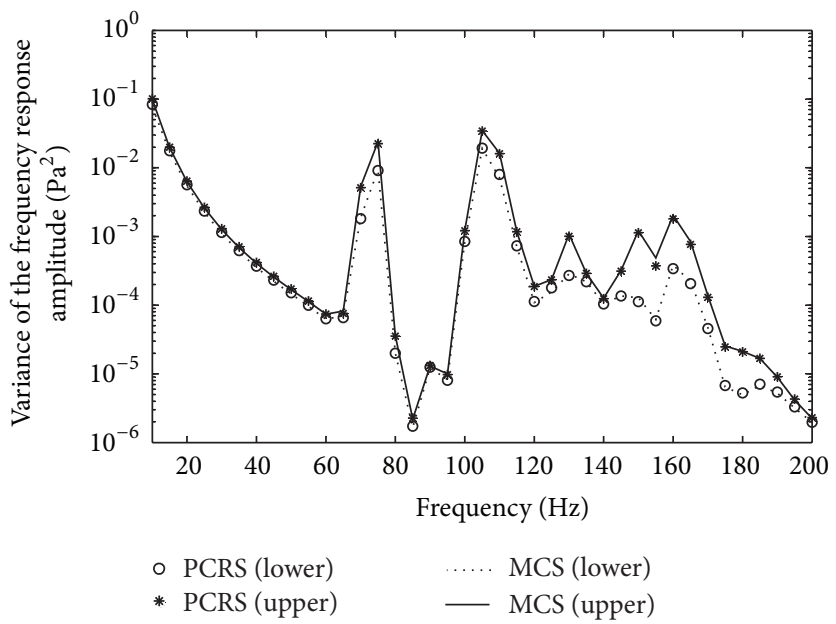

FIGURE 13: The lower and upper bounds of variance of the frequency response amplitude at node $R 1$ calculated by PCRS and MCS in the frequency band $f=10 \sim 200 \mathrm{~Hz}$.

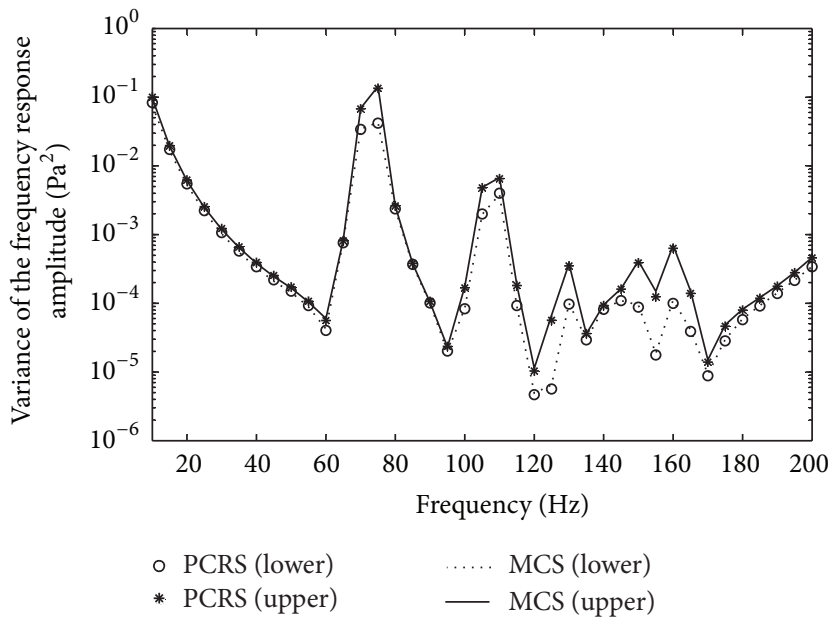

FIGURE 14: The lower and upper bounds of variance of the frequency response amplitude at node 22 calculated by PCRS and MCS in the frequency band $f=10 \sim 200 \mathrm{~Hz}$.

the lower and upper bounds of expectation and variance of response of the acoustic field with hybrid parameters can be efficiently evaluated by the interval analysis method. It is noted that this method is a nonintrusive method, in the sense that no modification to the deterministic governing system equations is required, which can be employed to solve black-box-type problems. The proposed method can also be applied to the other fields, such as structural-acoustic field, structure dynamic field, reliability, and robust design field and optimization field.

Two numerical examples are used to evaluate the performance of the PCRS method. The direct MCS method based on the original numerical method is implemented to validate the results obtained by the proposed method. The numerical results show that the PCRS method solutions match well with those yielded by the direct MCS method. Furthermore, the computational effort of the PCRS method is significantly lower than that of the direct MCS method.

However, it should be pointed out that the proposed method has its own limitations. The efficiency of the proposed method decreases as the number of random variables increases. To overcome this limitation, some efficient polynomial chaos methods can be introduced as a substitute for the generalized polynomial chaos in our future research. Additionally, in order to achieve more accurate results for the uncertain problems with hybrid uncertainties, other optimization methods or subinterval theory can be employed, which is our ongoing research.

\section{Competing Interests}

The authors declare that they have no competing interests. 


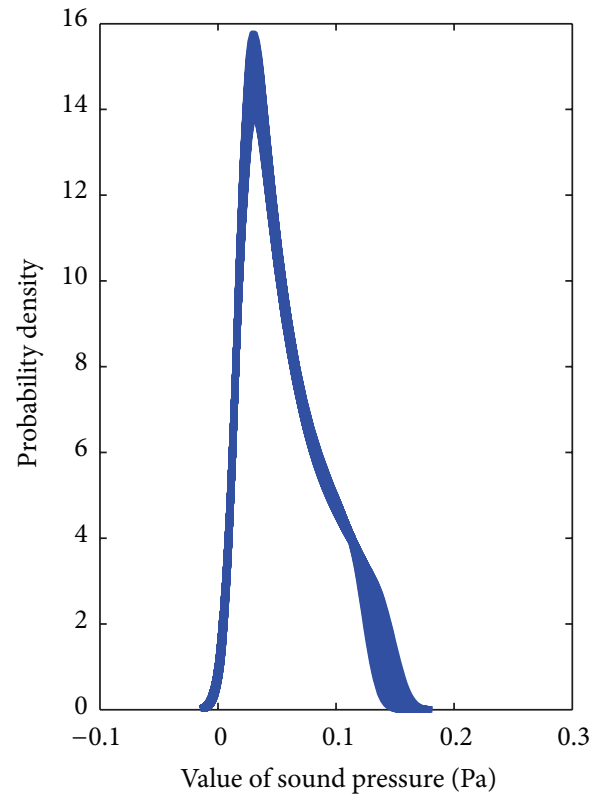

(a)

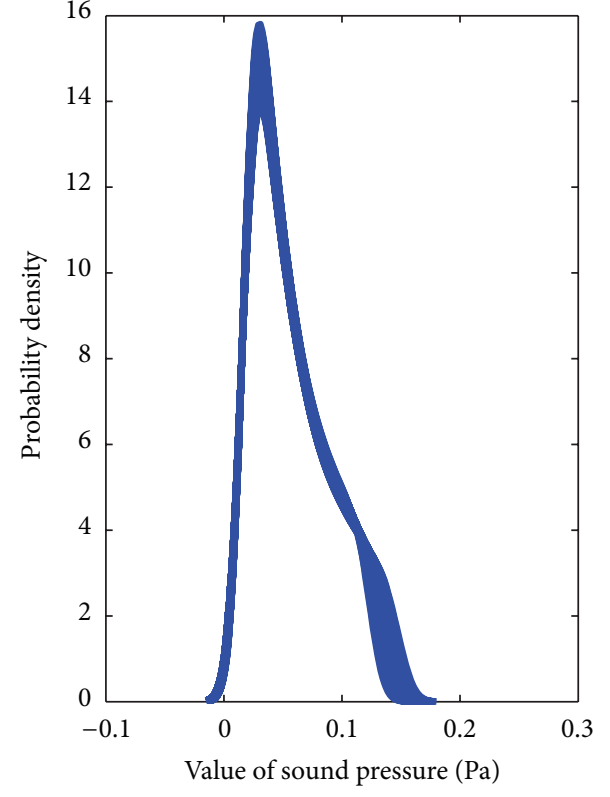

(b)

FIGURE 15: The lower and upper bounds of the response probability density of node $R 1$ calculated by (a) MCS and (b) PCRS.

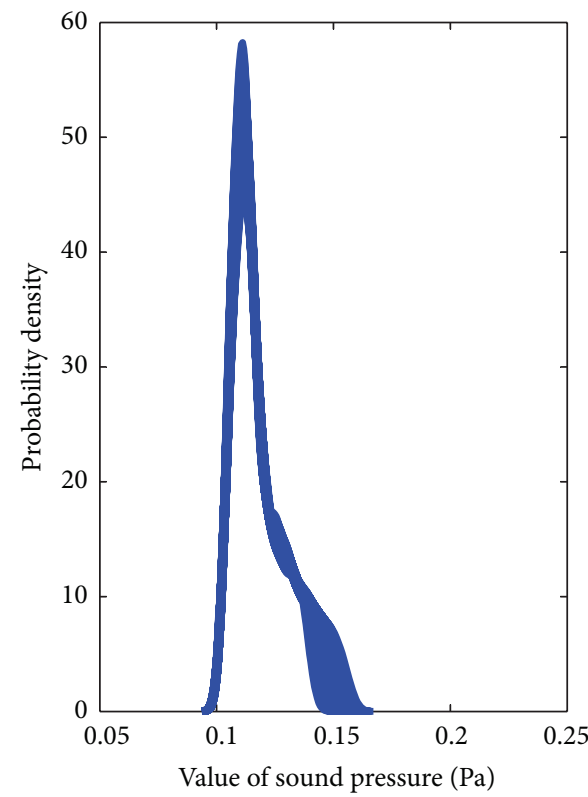

(a)

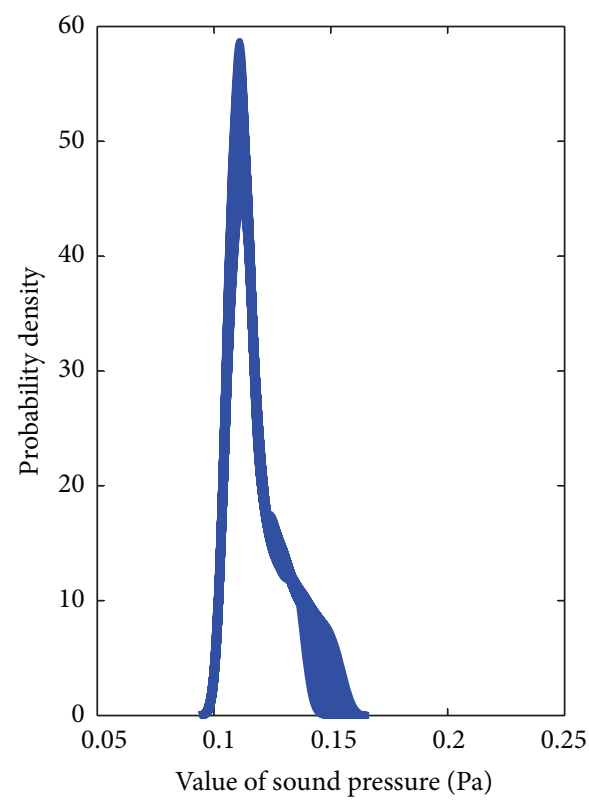

(b)

FIGURE 16: The lower and upper bounds of the response probability density of node $R 2$ calculated by (a) MCS and (b) PCRS.

\section{Acknowledgments}

Financial support from the National Nature Science Foundation of China (no. 51575201) is gratefully acknowledged.

\section{References}

[1] P. D. Spanos and A. Kontsos, "A multiscale Monte Carlo finite element method for determining mechanical properties of polymer nanocomposites," Probabilistic Engineering Mechanics, vol. 23, no. 4, pp. 456-470, 2008.

[2] S. Edgecombe and P. Linse, "Monte Carlo simulation of two interpenetrating polymer networks: structure, swelling, and mechanical properties," Polymer, vol. 49, no. 7, pp. 1981-1992, 2008.

[3] M. Kamiński, "Application of the generalized perturbationbased stochastic boundary element method to the elastostatics," 
Engineering Analysis with Boundary Elements, vol. 31, no. 6, pp. 514-527, 2007.

[4] G. I. Schuëller, "Developments in stochastic structural mechanics," Archive of Applied Mechanics, vol. 75, no. 10-12, pp. 755-773, 2006.

[5] A. Sarkar and R. Ghanem, "Mid-frequency structural dynamics with parameter uncertainty," Computer Methods in Applied Mechanics and Engineering, vol. 191, no. 47-48, pp. 5499-5513, 2002.

[6] O. M. Knio and O. P. Le Maître, "Uncertainty propagation in CFD using polynomial chaos decomposition," Fluid Dynamics Research, vol. 38, no. 9, pp. 616-640, 2006.

[7] K. Sepahvand, M. Scheffler, and S. Marburg, "Uncertainty quantification in natural frequencies and radiated acoustic power of composite plates: analytical and experimental investigation," Applied Acoustics, vol. 87, pp. 23-29, 2015.

[8] K. D. LePage, "Estimation of acoustic propagation uncertainty through polynomial Chaos expansions," in Proceedings of the 9th International Conference on Information Fusion (FUSION '09), IEEE, Florence, Italy, July 2006.

[9] D. B. Creamer, "On using polynomial chaos for modeling uncertainty in acoustic propagation," The Journal of the Acoustical Society of America, vol. 119, no. 4, pp. 1979-1994, 2006.

[10] S. Finette, "Embedding uncertainty into ocean acoustic propagation models (L)," The Journal of the Acoustical Society of America, vol. 117, no. 3, pp. 997-1000, 2005.

[11] Z. K. Nagy and R. D. Braatz, "Distributional uncertainty analysis using power series and polynomial chaos expansions," Journal of Process Control, vol. 17, no. 3, pp. 229-240, 2007.

[12] S. K. Saha, K. Sepahvand, V. A. Matsagar, A. K. Jain, and S. Marburg, "Stochastic analysis of base-isolated liquid storage tanks with uncertain isolator parameters under random excitation," Engineering Structures, vol. 57, pp. 465-474, 2013.

[13] D. Xiu and G. E. Karniadakis, "The Wiener-Askey polynomial chaos for stochastic differential equations," SIAM Journal on Scientific Computing, vol. 24, no. 2, pp. 619-644, 2002.

[14] D. Xiu and G. E. Karniadakis, "Modeling uncertainty in flow simulations via generalized polynomial chaos," Journal of Computational Physics, vol. 187, no. 1, pp. 137-167, 2003.

[15] K. Sepahvand, S. Marburg, and H.-J. Hardtke, "Uncertainty quantification in stochastic systems using polynomial chaos expansion," International Journal of Applied Mechanics, vol. 2, no. 2, pp. 305-353, 2010.

[16] K. Sepahvand, S. Marburg, and H.-J. Hardtke, "Stochastic free vibration of orthotropic plates using generalized polynomial chaos expansion," Journal of Sound and Vibration, vol. 331, no. 1, pp. 167-179, 2012.

[17] R. E. Moore, Interval Analysis, Prentice-Hall, Englewood Cliffs, NJ, USA, 1966.

[18] G. Alefeld and J. Herzberger, Introduction to Interval Computation, Academic Press, New York, NY, USA, 2012.

[19] S. Chen, H. Lian, and X. Yang, "Interval static displacement analysis for structures with interval parameters," International Journal for Numerical Methods in Engineering, vol. 53, no. 2, pp. 393-407, 2002.

[20] H. U. Köylüoglu and I. Elishakoff, "A comparison of stochastic and interval finite elements applied to shear frames with uncertain stiffness properties," Computers \& Structures, vol. 67, no. 1-3, pp. 91-98, 1998.
[21] B. Xia and D. Yu, "Modified interval perturbation finite element method for a structural-acoustic system with interval parameters," Journal of Applied Mechanics, vol. 80, no. 4, Article ID 041027, 2013.

[22] B. Xia, D. Yu, and J. Liu, "Interval and subinterval perturbation methods for a structural-acoustic system with interval parameters," Journal of Fluids and Structures, vol. 38, pp. 146-163, 2013.

[23] R. L. Muhanna, H. Zhang, and R. L. Mullen, "Interval finite elements as a basis for generalized models of uncertainty in engineering mechanics," Reliable Computing, vol. 13, no. 2, pp. 173-194, 2007.

[24] S.-E. Fang, Q.-H. Zhang, and W.-X. Ren, "An interval model updating strategy using interval response surface models," Mechanical Systems and Signal Processing, vol. 60, pp. 909-927, 2015.

[25] T. Zou, M. Cai, R. Du, and J. Liu, "Analyzing the uncertainty of simulation results in accident reconstruction with Response Surface Methodology," Forensic Science International, vol. 216, no. 1-3, pp. 49-60, 2012.

[26] J. Guo and X. Du, "Reliability sensitivity analysis with random and interval variables," International Journal for Numerical Methods in Engineering, vol. 78, no. 13, pp. 1585-1617, 2009.

[27] W. Gao, D. Wu, C. Song, F. Tin-Loi, and X. Li, "Hybrid probabilistic interval analysis of bar structures with uncertainty using a mixed perturbation Monte-Carlo method," Finite Elements in Analysis and Design, vol. 47, no. 7, pp. 643-652, 2011.

[28] B. Xia, D. Yu, and J. Liu, "Probabilistic interval perturbation methods for hybrid uncertain acoustic field prediction," Journal of Vibration and Acoustics, vol. 135, no. 2, Article ID 021009, 2013.

[29] B. Xia and D. Yu, "Response probability analysis of random acoustic field based on perturbation stochastic method and change-of-variable technique," Journal of Vibration and Acoustics, Transactions of the ASME, vol. 135, no. 5, Article ID 051032, 2013.

[30] B. Xia and D. Yu, "Modified sub-interval perturbation finite element method for 2D acoustic field prediction with large uncertain-but-bounded parameters," Journal of Sound and Vibration, vol. 331, no. 16, pp. 3774-3790, 2012.

[31] C. Wang and Z. Qiu, "An interval perturbation method for exterior acoustic field prediction with uncertain-but-bounded parameters," Journal of Fluids and Structures, vol. 49, pp. 441449, 2014.

[32] B. Xia and D. Yu, "Interval analysis of acoustic field with uncertain-but-bounded parameters," Computers and Structures, vol. 112-113, pp. 235-244, 2012.

[33] B. Xia and D. Yu, "Response analysis of acoustic field with convex parameters," Journal of Vibration and Acoustics, vol. 136, no. 4, Article ID 041017, 2014.

[34] B. Xia, D. Yu, and J. Liu, "Hybrid uncertain analysis for structural-acoustic problem with random and interval parameters," Journal of Sound and Vibration, vol. 332, no. 11, pp. 27012720, 2013.

[35] B. Xia, S. Yin, and D. Yu, "A new random interval method for response analysis of structural-acoustic system with interval random variables," Applied Acoustics, vol. 99, pp. 31-42, 2015.

[36] B. Xia, D. Yu, and J. Liu, "Hybrid uncertain analysis of acoustic field with interval random parameters," Computer Methods in Applied Mechanics and Engineering, vol. 256, pp. 56-69, 2013.

[37] N. Wiener, "The homogeneous chaos," American Journal of Mathematics, vol. 60, no. 4, pp. 897-936, 1938. 
[38] A. Sandu, C. Sandu, and M. Ahmadian, "Modeling multibody systems with uncertainties. Part I: theoretical and computational aspects," Multibody System Dynamics, vol. 15, no. 4, pp. 369-391, 2006.

[39] M. S. Eldred, "Recent advances in non-intrusive polynomial chaos and stochastic collocation methods for uncertainty analysis and design," in Proceedings of the 50th AIAA/ASME/ASCE/ AHS/ASC Structures, Structural Dynamics, and Materials Conference, AIAA Paper 2009-2274, Palm Springs, Calif, USA, 2009.

[40] O. Bonin, "Sensitivity analysis and uncertainty analysis for vector geographical applications," in Proceedings of the 7th International Symposium on Spatial Accuracy Assessment in Natural Resources and Environmental Sciences (ACCURACY '06), Lisbon, Portugal, July 2006.

[41] X. Qu, Reliability-Based Structural Optimization Using Response Surface Approximations and Probabilistic Sufficiency Factor, University of Florida, 2004.

[42] M. Cai, T. Zou, P. Luo, and J. Li, "Evaluation of simulation uncertainty in accident reconstruction via combining Response Surface Methodology and Monte Carlo Method," Transportation Research Part C: Emerging Technologies, vol. 48, pp. 241-255, 2014.

[43] H. H. Khodaparast, J. E. Mottershead, and K. J. Badcock, "Interval model updating with irreducible uncertainty using the Kriging predictor," Mechanical Systems and Signal Processing, vol. 25, no. 4, pp. 1204-1226, 2011.

[44] R. Anderssohn and S. Marburg, "Nonlinear approach to approximate acoustic boundary admittance in cavities," Journal of Computational Acoustics, vol. 15, no. 1, pp. 63-79, 2007.

[45] Z. Wan, T. Wang, Q. Huang, and J. Wang, "Acoustic finite element model updating using acoustic frequency response function," Finite Elements in Analysis and Design, vol. 87, pp. 1-9, 2014. 


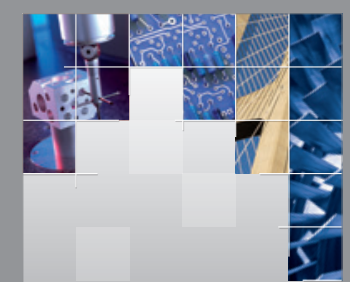

\section{Enfincering}
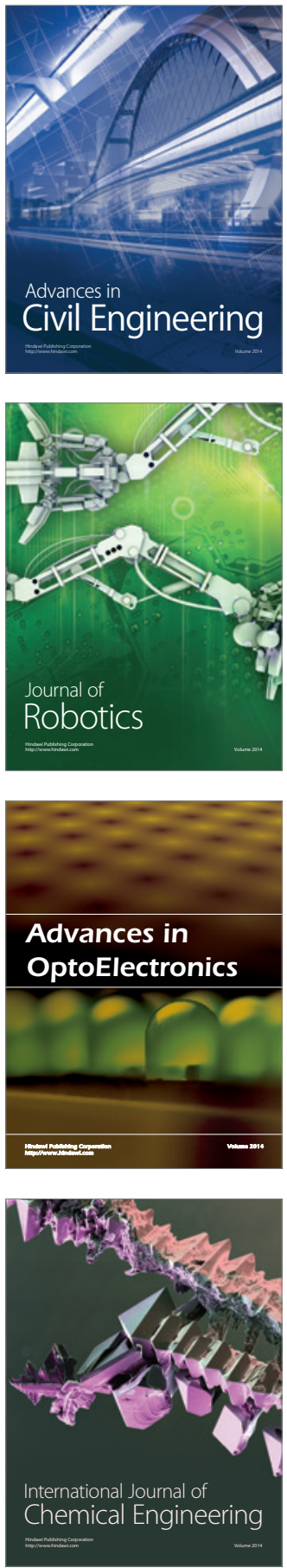

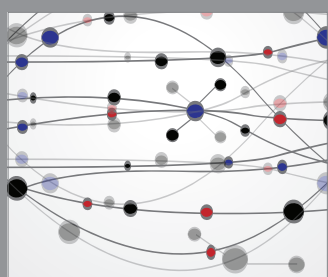

The Scientific World Journal

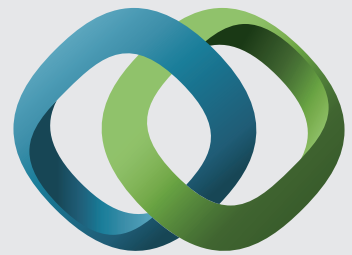

\section{Hindawi}

Submit your manuscripts at

http://www.hindawi.com
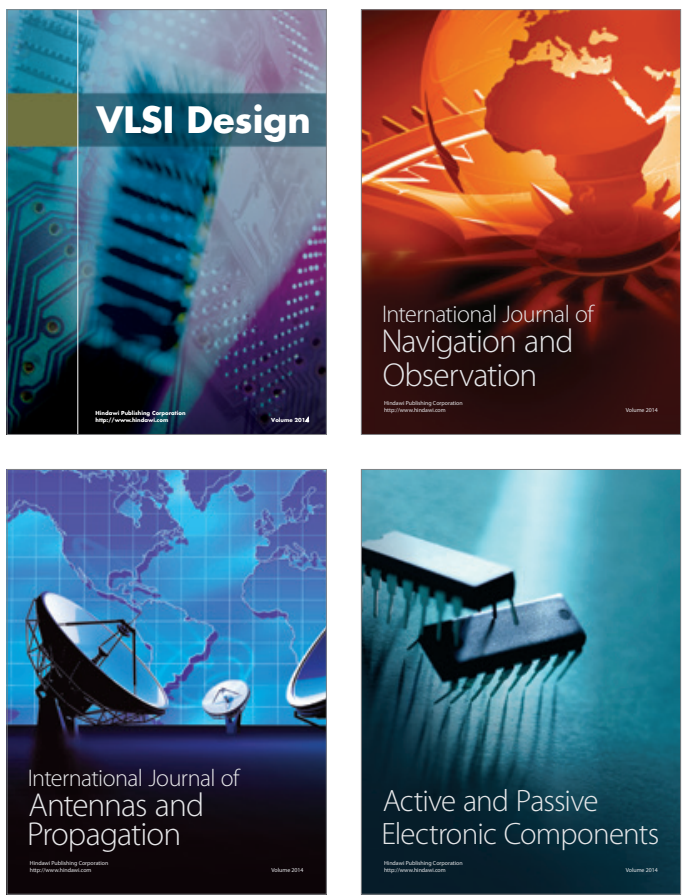
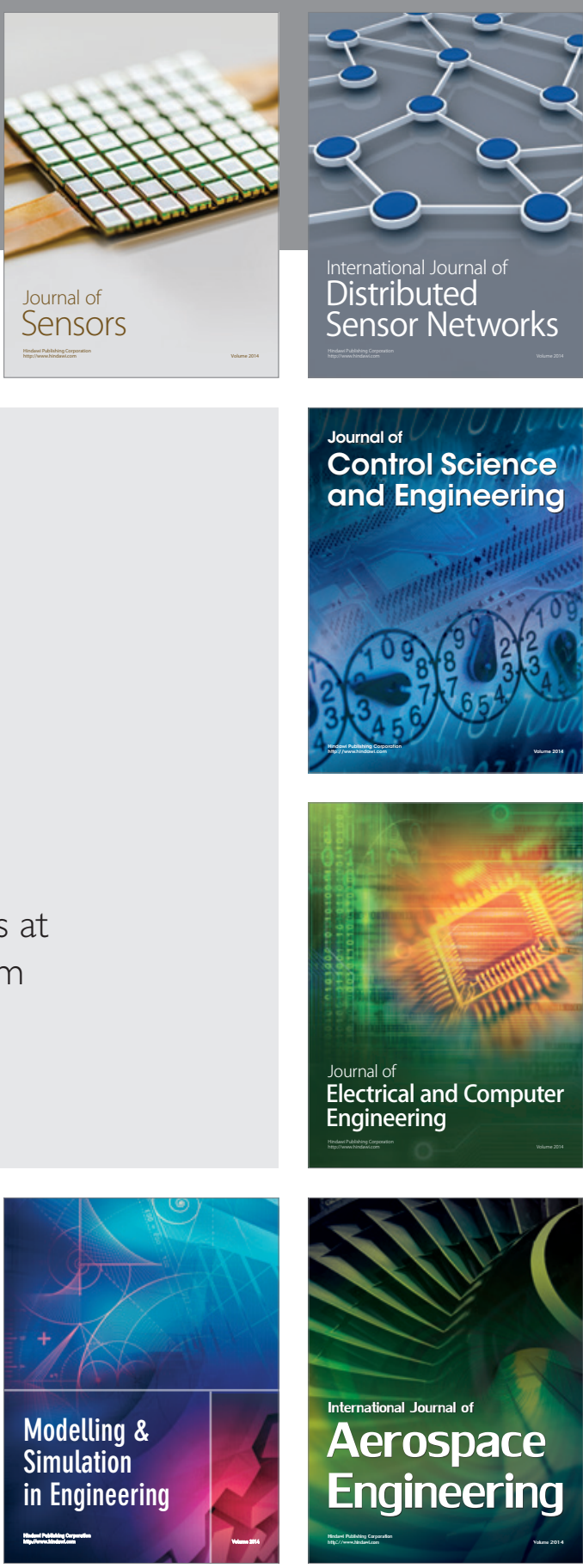

International Journal of

Distributed

Sensor Networks

Journal of

Control Science

and Engineering
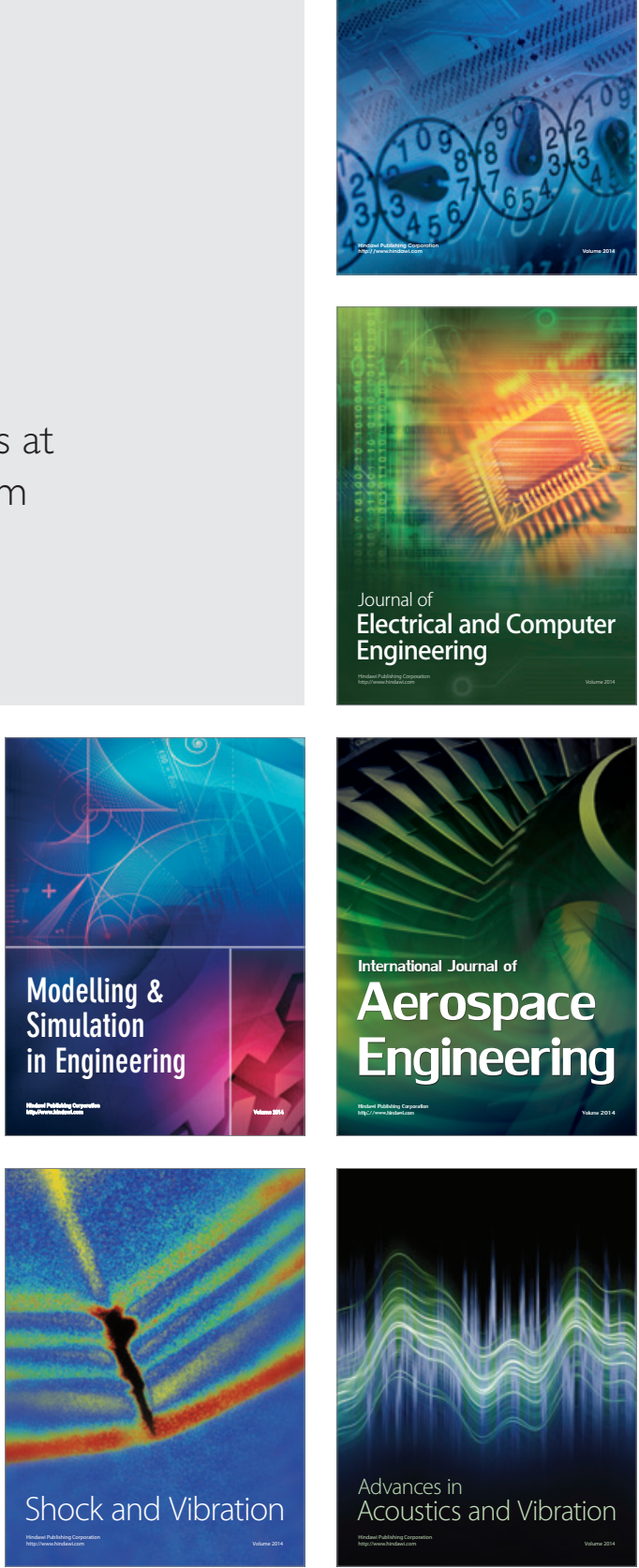